TRANSACTIONS OF THE

AMERICAN MATHEMATICAL SOCIETY

Volume 359, Number 3, March 2007, Pages 965-990

S 0002-9947(06)04094-3

Article electronically published on October 16, 2006

\title{
THE CLASSIFICATION OF SINGLY PERIODIC MINIMAL SURFACES WITH GENUS ZERO AND SCHERK-TYPE ENDS
}

\author{
JOAQUÍN PÉREZ AND MARTIN TRAIZET
}

\begin{abstract}
Given an integer $k \geq 2$, let $\mathcal{S}(k)$ be the space of complete embedded singly periodic minimal surfaces in $\mathbb{R}^{3}$, which in the quotient have genus zero and $2 k$ Scherk-type ends. Surfaces in $\mathcal{S}(k)$ can be proven to be proper, a condition under which the asymptotic geometry of the surfaces is well known. It is also known that $\mathcal{S}(2)$ consists of the 1-parameter family of singly periodic Scherk minimal surfaces. We prove that for each $k \geq 3$, there exists a natural one-to-one correspondence between $\mathcal{S}(k)$ and the space of convex unitary nonspecial polygons through the map which assigns to each $M \in \mathcal{S}(k)$ the polygon whose edges are the flux vectors at the ends of $M$ (a special polygon is a parallelogram with two sides of length 1 and two sides of length $k-1$ ). As consequence, $\mathcal{S}(k)$ reduces to the saddle towers constructed by Karcher.
\end{abstract}

\section{INTRODUCTION}

In 1834, Scherk 18 discovered a 1-parameter family of singly periodic, complete, properly embedded minimal surfaces in Euclidean space $\mathbb{R}^{3}$. Each of these surfaces may be seen as the desingularisation of two vertical planes: the angle $\theta \in(0, \pi / 2]$ between the planes is the parameter of the family. These surfaces are now called Scherk singly periodic minimal surfaces. In the quotient $\mathbb{R}^{3} / T$ by the shortest orientation-preserving translation $T$, these surfaces have genus zero and four ends asymptotic to flat vertical annuli (namely the quotients of vertical half-planes by $T)$. Such ends are called Scherk-type ends.

In 1988, Karcher [5] constructed for each integer $k \geq 3$ a $(2 k-3)$-parameter family of properly embedded singly periodic minimal surfaces which have $2 k$ Scherktype ends and genus zero in the quotient by the period. These surfaces are now called Karcher saddle towers.

The main result of this paper is

Theorem 1.1. Let $M$ be a complete embedded singly periodic minimal surface in $\mathbb{R}^{3}$ with a finite number of Scherk-type ends and genus zero in the quotient by the period. Then $M$ is a singly periodic Scherk surface or a Karcher saddle tower.

An interesting problem is to classify all complete embedded minimal surfaces in complete flat 3-manifolds, which have genus zero and finite topology (namely, a finite number of ends). An important property of these surfaces is that they are

Received by the editors September 29, 2004.

2000 Mathematics Subject Classification. Primary 53A10; Secondary 49Q05, 53C42.

Key words and phrases. Singly periodic minimal surface, Scherk-type end, moduli space.

The research of the first author was partially supported by a MEC/FEDER grant no. MTM2004-02746.

(C)2006 American Mathematical Society Reverts to public domain 28 years from publication 
always proper, as follows from Proposition 1.3 below. The above theorem allows us to complete the classification in the case of the flat manifold $\mathbb{R}^{2} \times \mathbb{S}^{1}=\mathbb{R}^{3} / T$. Indeed, by a theorem of Meeks and Rosenberg [11, in this case all ends must be simultaneously asymptotic to horizontal planes, helicoids or vertical halfplanes (Scherk-type ends). The first case when $M$ has genus zero leads to a plane by the maximum principle for minimal surfaces. In the second case, the only example is the helicoid by a theorem of Pérez and Ros 14. Therefore we obtain the following result.

Corollary 1.2. Let $M$ be a nonflat complete embedded minimal surface in $\mathbb{R}^{2} \times \mathbb{S}^{1}$. Assume $M$ has genus zero and finite topology. Then $M$ is a helicoid, a singly periodic Scherk minimal surface, or a Karcher saddle tower.

The classification of the complete embedded nonflat minimal planar domains with finitely many ends is also complete in the following ambient spaces: In $\mathbb{R}^{3}$, $M$ must be a catenoid (Collin [1, López and Ros [7]) or a helicoid (Meeks and Rosenberg [12]). In $\mathbb{T}^{2} \times \mathbb{R}, M$ must be a doubly periodic Scherk surface (LazardHolly and Meeks [6]). In $\mathbb{T}^{3}$ there are no examples, since $M$ must have genus at least 3 (Meeks [8]). The only case that remains open is the flat manifold $\mathbb{R}^{3} / S_{\theta}$ where $S_{\theta}$ is a screw motion.

We mentioned above that the classification of complete embedded minimal surfaces of genus zero in complete flat 3-manifolds can be reduced to the proper ones. This reduction is an immediate consequence of arguments in the proof of the recent minimal lamination closure theorem by Meeks and Rosenberg [10].

Proposition 1.3. Let $M \subset N^{3}$ be a complete embedded minimal surface with finite topology in a complete flat 3-manifold. Then, $M$ is proper.

For the sake of completeness, we briefly sketch a proof of Proposition 1.3. We claim that $M$ has positive injectivity radius. Otherwise, there exists a divergent sequence $\gamma_{n}$ of closed geodesics of $M$, each one smooth except at one point, with lengths tending to zero. By the Gauss-Bonnet formula, $\gamma_{n}$ cannot bound a disk on $M$. Since $M$ has finite topology and $\left\{\gamma_{n}\right\}_{n}$ diverges, we can assume (after passing to a subsequence) that the $\gamma_{n}$ all lie in an annular end $E$ of $M$. Applying again the Gauss-Bonnet formula to the compact annulus bounded by $\gamma_{1}$ and $\gamma_{n}$ and then taking limits, one easily concludes that $E$ has finite total curvature. Since $E$ is complete and embedded, it must be asymptotic to a plane, a half-catenoid, a flat annulus or to the end of a helicoid. But all these ends have positive or infinite injectivity radius, which is a contradiction. Hence, $M$ has positive injectivity radius, and the same holds for the periodic minimal surface $\widetilde{M} \subset \mathbb{R}^{3}$ produced by lifting $M$. By the minimal lamination closure theorem, $\widetilde{M}$ is proper in $\mathbb{R}^{3}$. Since it is also periodic, the induced surface $M$ is proper as well, which finishes the proof.

The proof of Theorem 1.1 is a modified application of the machinery developed by Meeks, Pérez and Ros in their characterization of Riemann minimal examples 9]. It is known [11 that $M$ must have an even number $2 k \geq 4$ of ends (this is a simple consequence of embeddedness). For $k \geq 2$, let $\mathcal{S}(k)$ be the space of properly embedded singly periodic minimal surfaces with genus zero and $2 k$ Scherk-type ends. In the case $k=2$, it is elementary to see that $\mathcal{S}(2)$ reduces to the family of Scherk singly periodic surfaces. When $k \geq 3$, the goal is to prove that $\mathcal{S}(k)$ reduces to the space $\mathcal{K}(k)$ of Karcher saddle towers with $2 k$ ends. The argument is based on modeling $\mathcal{S}(k)$ as an analytical subset in a complex manifold $\mathcal{W}(k)$ of 
finite dimension (roughly, $\mathcal{W}(k)$ consists of all admissible Weierstrass data for our problem). Then the procedure has three steps:

- Properness: Uniform curvature estimates are proven for a sequence of surfaces in $\mathcal{S}(k)$, provided one has some control on the flux at the ends.

- Openness: Any surface in $\mathcal{S}(k) \backslash \mathcal{K}(k)$ may be deformed into another surface in $\mathcal{S}(k) \backslash \mathcal{K}(k)$ by locally perturbing the flux at the ends. Together with the properness property, this implies that if $\mathcal{S}(k) \backslash \mathcal{K}(k)$ is nonempty, then any configuration of flux may be achieved by surfaces in $\mathcal{S}(k) \backslash \mathcal{K}(k)$.

- Local uniqueness: We prove that some particular configuration of fluxes may only be achieved by surfaces in $\mathcal{K}(k)$. This proves that $\mathcal{S}(k) \backslash \mathcal{K}(k)$ is empty.

The paper is organized as follows. In Section 2 we recall the necessary background for our problem. Furthermore, we provide an elementary proof of the case $k=2$, we remind the construction of the Karcher saddle towers and we define the flux map, which is the main tool to prove our main theorem. In Section 3 we study the space of admissible Weierstrass data. In Section 4 we prove our main theorem assuming the properness, openness and local uniqueness statements. Properness is studied in Section 5 and openness in Section 6. In Sections 7 and 8 we prove local uniqueness in a neighborhood of some limit cases.

\section{Preliminaries}

Let $\widetilde{M} \subset \mathbb{R}^{3}$ be a complete embedded minimal surface invariant by the translation of vector $T=(0,0,2 \pi)$. $\widetilde{M}$ induces a complete embedded minimal surface $M=\widetilde{M} / T \subset \mathbb{R}^{3} / T=\mathbb{R}^{2} \times \mathbb{S}^{1}$. From now on, assume that $M$ has finite topology (hence, it is proper by Proposition 1.3). Meeks and Rosenberg [11 proved that $M$ has finite total curvature, and so $M$ is conformally a finitely punctured closed Riemann surface. Furthermore, $M$ has an even number of ends, all of them simultaneously asymptotic to nonvertical planes, vertical helicoids or flat vertical annuli. These asymptotic behaviors are called respectively planar, helicoidal or Scherk-type ends. In the sequel we will assume that $M$ has genus zero and Scherk-type ends.

For a fixed integer $k \geq 2$, we will denote by $\mathcal{S}(k)$ the space of properly embedded singly periodic minimal surfaces which are invariant by the translation $T=(0,0,2 \pi)$, with genus zero in the quotient and $2 k$ Scherk-type ends, modulo translations and rotations around the $x_{3}$-axis. $\mathcal{S}(k)$ can be naturally endowed with the uniform topology on compact sets of $\mathbb{R}^{3} / T$. Any surface $M \in \mathcal{S}(k)$ is conformally equivalent to the Riemann sphere $\overline{\mathbb{C}}=\mathbb{C} \cup\{\infty\}$ minus $2 k$ points $p_{1}, \ldots, p_{2 k}$ that correspond to the ends of $M$. The Gauss map (stereographically projected) $g$ of the singly periodic lifting $\widetilde{M} \subset \mathbb{R}^{3}$ of $M$ descends to the quotient surface, giving rise to a meromorphic map on $M$ that extends holomorphically through each puncture (with $\left|g\left(p_{j}\right)\right|=1$ because the ends are asymptotic to flat vertical annuli). The degree of such an extension $g: \overline{\mathbb{C}} \rightarrow \overline{\mathbb{C}}$ is given by the Meeks-Rosenberg formula [1], which in this setting writes

$$
\operatorname{deg}(g)=k-1 .
$$

The height differential $\phi=\frac{\partial x_{3}}{\partial z} d z$, where $x_{3}$ is the third coordinate function on $\widetilde{M}$ and $z$ is a local conformal coordinate, also descends to a meromorphic differential on $M$ which extends through each $p_{j}$ having a simple pole with residue $\pm i$ (this comes from the normalization of the period vector to be $\pm T$ ). Hence the flux vector 
$F_{j}$ at the end $p_{j}$, defined as the integral of the inner unit conormal vector to $M$ along the boundary of an end representative of $p_{j}$, is given by

$$
F_{j}=2 \pi u_{j}
$$

where $u_{j}$ is a unitary horizontal vector in $\mathbb{R}^{3}$ (in fact, $u_{j}$ is orthogonal to the limit normal vector at $p_{j}$ ). The divergence theorem implies that

$$
\sum_{j=1}^{2 k} u_{j}=0
$$

For the remainder of the paper, we will use the identification $\mathbb{R}^{3} \equiv \mathbb{C} \times \mathbb{R}$ given by $(a, b, c) \equiv(a+i b, c)$. Hence, we can write $u_{j}=e^{i \theta_{j}} \in \mathbb{S}^{1}=\{|z|=1\} \subset \mathbb{C}$ with $\theta_{i} \in \mathbb{R}$.

The goal of this paper is to classify all elements in $\mathcal{S}(k)$ for each $k \geq 3$. Although the case $k=2$ is well known, we include an elementary proof of the description of $\mathcal{S}(2)$ for the sake of completeness.

Lemma 2.1. $\mathcal{S}(2)$ consists of the 1-parameter family of singly periodic Scherk surfaces.

Proof. Fix $M \in \mathcal{S}(2)$, with ends $p_{1}, p_{2}, p_{3}, p_{4}$ cyclically ordered. Using (2.2) and (2.3), it is easy to check that $F_{1}=-F_{3}$ and $F_{2}=-F_{4}$. This implies that, up to a rotation around the $x_{3}$-axis, the values at the ends of the stereographically projected extended Gauss map $g$ of $M$ are $g\left(p_{1}\right)=e^{i \theta}, g\left(p_{2}\right)=e^{-i \theta}, g\left(p_{3}\right)=-g\left(p_{1}\right)$ and $g\left(p_{4}\right)=-g\left(p_{2}\right)$ for certain $\theta \in(0, \pi / 2)$. Since (2.1) says that $\operatorname{deg}(g)=1$, we can parametrize $M$ by its Gauss map, i.e. $M=\overline{\mathbb{C}} \backslash\left\{ \pm e^{ \pm i \theta}\right\}$ with $g(z)=z$. As the height differential $\phi$ of $M$ has simple poles at $\pm e^{ \pm i \theta}$ and simple zeros at $0, \infty$ (because $0, \infty$ are finite points of $M$ ), we have

$$
\phi=c \frac{z d z}{\prod\left(z \pm e^{ \pm i \theta}\right)}
$$

where $c \in \mathbb{C}^{*}=\mathbb{C}-\{0\}$. Finally, as $\operatorname{Res}_{p_{j}} \phi= \pm i$ for each $j$ we deduce that $c=4 \sin (2 \theta)$, hence the Weierstrass data $(g, \phi)$ represent a singly periodic Scherk minimal surface.

For the remainder of the paper we will only deal with $2 k$-ended surfaces, $k \geq 3$. Given $M \in \mathcal{S}(k)$ with ends $p_{1}, \ldots, p_{2 k}$, there is a natural cyclic ordering on the set of ends so that the arguments $\theta_{j}$ of the flux vectors $F_{j}=2 \pi e^{i \theta_{j}}$ satisfy

$$
\theta_{1} \leq \theta_{2} \leq \cdots \leq \theta_{2 k} \leq \theta_{1}+2 \pi,
$$

but there is no natural choice for the first end $p_{1}$. The surface $M$ together with the choice of the first end $p_{1}$ is what we will call a marked surface (unless it leads to confusion, we will keep the notation $M$ for a marked surface). Let $\widetilde{\mathcal{S}}(k)$ be the set of such marked minimal surfaces, which naturally inherits the uniform topology on compact sets. Since our surfaces are defined up to rotations about the $x_{3}$-axis, we can assume that $F_{1}=1$ for each $M \in \widetilde{\mathcal{S}}(k)$.

Let $\mathcal{U}(k)$ be the set of marked convex $2 k$-gons in $\mathbb{C}$ with edges of unit length. By marked we mean that for each polygon we choose a vertex. To avoid translations and rotations, we normalize so that the points $z=0$ and $z=1$ are consecutive 
vertices of each element in $\mathcal{U}(k)$ and the chosen vertex is 0 . We will identify $\mathcal{U}(k)$ with the space

$$
\left\{\mathbf{u}=\left(u_{1}, \ldots, u_{2 k}\right)=\left(1, e^{i \theta_{2}}, \cdots, e^{i \theta_{2 k}}\right) \in\left(\mathbb{S}^{1}\right)^{2 k} \mid \text { (2.3) and (2.4) hold }\right\},
$$

so that a list $\mathbf{u}$ corresponds to the marked polygon $P_{\mathbf{u}}$ of consecutive vertices $Z_{1}=0$, $Z_{j}=Z_{j-1}+e^{i \theta_{j}}$ for $j=1, \ldots, 2 k . \mathcal{U}(k)$ can be seen as a subset of the space $\mathcal{V}(k)$ of unitary $2 k$-gons, not necessarily convex or embedded. $\mathcal{V}(k)$ has a natural structure of real analytic manifold of dimension $2 k-3$ (with the angles at consecutive vertices as local parameters). Since polygons in $\mathcal{U}(k)$ are convex but not necessarily strictly convex, $\mathcal{U}(k)$ is a closed subset of $\mathcal{V}(k)$. Clearly $\mathcal{U}(k)$ is connected, and its boundary consists of those convex $2 k$-gons with at least one edge of length 2 (viewed as two consecutive edges).

Definition 2.2. An element $\mathbf{u} \in \mathcal{U}(k)$ is said to be a special polygon if there exist $v, w \in \mathbb{S}^{1}$ so that two edges of $P_{\mathbf{u}}$ are equal to $\pm v$ and all other edges are equal to $\pm w$. In other words, $P_{\mathbf{u}}$ is a (possibly degenerated) parallelogram with two edges of length 1 and two edges of length $k-1$, these last ones considered as $k-1$ consecutive unitary edges of $P_{\mathbf{u}}$. We will denote by $\mathcal{U}_{0}(k)$ the subset of special polygons. The limit case $v= \pm w$ represents a parallelogram that degenerates in the segment with end points $0, k$. We will call $\mathbf{u}_{0}(k)$ this degenerate parallelogram.

Definition 2.3. With the notation above, we define the flux map $F: \widetilde{\mathcal{S}}(k) \rightarrow \mathcal{U}(k)$ as $M \in \widetilde{\mathcal{S}}(k) \mapsto F(M)=\mathbf{u}$, where $\mathbf{u}=\left(u_{1}, \ldots, u_{2 k}\right)$ is defined by (2.2). Since the $u_{j}$ are the flux vectors at the ends of $M$ (up to $\frac{1}{2 \pi}$ ), $F$ is clearly continuous. A marked surface $M \in \widetilde{\mathcal{S}}(k)$ is called special if its flux polygon $F(M)$ is special. We will denote by $\widetilde{\mathcal{S}}_{0}(k)=F^{-1}\left(\mathcal{U}_{0}(k)\right)$ the space of special marked surfaces.

Remark 2.4. A simple consequence of the maximum principle for minimal surfaces insures that $F^{-1}\left(\mathbf{u}_{0}(k)\right)=\varnothing$.

2.1. Karcher saddle towers. We now recall how to construct a surface $M \in \widetilde{\mathcal{S}}(k)$ from a given nonspecial $2 k$-gon (for details, see Karcher [5]). Given $\mathbf{u} \in \mathcal{U}(k) \backslash \mathcal{U}_{0}(k)$, a theorem by Jenkins-Serrin [4] insures that there exists a minimal graph $G_{\mathbf{u}}$ with boundary values alternately $+\infty$ and $-\infty$ on the sides of the polygon $P_{\mathbf{u}}$ (which we see inside the plane $\{z=0\} \subset \mathbb{R}^{3}$ ). Since $G_{\mathbf{u}}$ is bounded by vertical lines over the vertices of $P_{\mathbf{u}}$, the conjugate surface $G_{\mathbf{u}}^{*}$ of $G_{\mathbf{u}}$ is bounded by horizontal arcs which lie alternately in two horizontal planes. $G_{\mathbf{u}}^{*}$ can be extended by reflection in such horizontal arcs to a surface $M_{\mathbf{u}} \in \mathcal{S}(k)$ called a Karcher saddle tower, whose flux polygon is $\mathbf{u}$. Since $\mathcal{U}(k)$ has $2 k-3$ freedom parameters, we deduce that the Karcher saddle towers come in a $(2 k-3)$-parameter family $\mathcal{K}(k)$ of examples.

Since each surface $M_{\mathbf{u}} \in \mathcal{K}(k)$ has genus zero and admits a reflective symmetry $R_{\Pi}$ across a horizontal plane $\Pi$ such that the set of fixed points of $R_{\Pi}$ coincides with $M_{\mathbf{u}} \cap \Pi$, Theorem 1 in Cosín and Ros [2] insures that the only bounded Jacobi functions on $M_{\mathbf{u}}$ are linear functions of its Gauss map. This condition and an implicit function theorem argument (see for instance Pérez and Ros [15]) imply that $\mathcal{K}(k)$ is open in $\mathcal{S}(k)$. By construction, $\mathcal{K}(k)$ is also closed in $\mathcal{S}(k)$. The continuous dependence on the $2 k$-gon $\mathbf{u}$ of the Jenkins-Serrin graph $G_{\mathbf{u}}$ gives that the map $\mathbf{u} \in \mathcal{U}(k) \backslash \mathcal{U}_{0}(k) \mapsto M_{\mathbf{u}} \in \widetilde{\mathcal{S}}(k)$ is continuous. Since $\mathcal{U}(k) \backslash \mathcal{U}_{0}(k)$ is connected, we deduce that $\mathcal{K}(k)$ forms a component of $\mathcal{S}(k)$. Similarly as with other spaces of surfaces, $\widetilde{\mathcal{K}}(k)$ will stand for the space of marked Karcher saddle towers, which is open and closed in $\widetilde{\mathcal{S}}(k)$. 
Remark 2.5. The hypothesis of the Jenkins-Serrin theorem is as follows: for each strict sub-polygon $Q$ of $P_{\mathbf{u}}$ (which means that the vertices of $Q$ form a strict subset of those of $P_{\mathbf{u}}$ ), the perimeter of $Q$ must be strictly larger than twice the number of edges of $Q \cap P_{\mathbf{u}}$ marked with $+\infty$ (resp. $-\infty$ ). While this hypothesis is satisfied by any polygon in $\mathcal{U}(k) \backslash \mathcal{U}_{0}(k)$, it fails to be true for special polygons: simply consider any sub-rhombus of a special polygon $\mathbf{u} \in \mathcal{U}_{0}(k)$ (we thank Barbara Nelli for pointing out this fact to us).

Another consequence of the continuity of the map $\mathbf{u} \in \mathcal{U}(k) \backslash \mathcal{U}_{0}(k) \mapsto M_{\mathbf{u}} \in \widetilde{\mathcal{S}}(k)$ is that we can equivalently state Lemma 2.1 by saying that $F: \widetilde{\mathcal{S}}(2) \rightarrow \mathcal{U}(2) \backslash$ $\left\{\mathbf{u}_{0}(2)\right\}$ is a homeomorphism. Our main theorem is now stated as follows.

Theorem 2.6. Assume $k \geq 3$. Then $\mathcal{S}(k)=\mathcal{K}(k)$ and the map $F: \widetilde{\mathcal{S}}(k) \rightarrow$ $\mathcal{U}(k) \backslash \mathcal{U}_{0}(k)$ is a homeomorphism, whose inverse map is $u \mapsto M_{\mathbf{u}}$ (in particular, $\left.\widetilde{\mathcal{S}}_{0}(k)=\varnothing\right)$.

Since each Karcher saddle tower $M_{\mathbf{u}}$ is obtained by symmetrization of the conjugate graph $G_{\mathbf{u}}^{*}$ across the horizontal planes containing $\partial G_{\mathbf{u}}^{*}$, we directly obtain the following corollary.

Corollary 2.7. Any properly embedded singly periodic minimal surface with genus zero and a finite number of Scherk-type ends has a horizontal plane of symmetry.

\section{The SPACE of Weierstrass Representations}

Let $M \in \widetilde{\mathcal{S}}(k)$ be an embedded marked surface with ends $p_{1}, \ldots, p_{2 k}$, flux polygon $F(M)=\mathbf{u}=\left(u_{1}, \ldots, u_{2 k}\right)$, complex Gauss map $g$ and height differential $\phi$. The limit normal values are $g\left(p_{j}\right)= \pm i u_{j}, 1 \leq j \leq 2 k$. Since $M$ is embedded, the normal vector always points to the same component of $\left(\mathbb{R}^{3} / T\right) \backslash M$, hence we may assume without loss of generality that

$$
g\left(p_{j}\right)=(-1)^{j+1} i u_{j},
$$

and thus the flux at the end $p_{j}$ is given in terms of $(g, \phi)$ by

$$
F_{j}=2 \pi u_{j}=-2 \pi g\left(p_{j}\right) \operatorname{Res}_{p_{j}} \phi=2 \pi(-1)^{j} i u_{j} \operatorname{Res}_{p_{j}} \phi,
$$

which yields

$$
\operatorname{Res}_{p_{j}} \phi=(-1)^{j} i, \quad 1 \leq j \leq 2 k .
$$

Let $\mathcal{W}(k)$ be the set of lists $\left(g, p_{1}, \ldots, p_{2 k}\right)$ where $g$ is a degree $k-1$ meromorphic function on $\overline{\mathbb{C}}$ and $p_{1}, \ldots, p_{2 k} \in \mathbb{C}^{*}$ are $2 k$ distinct points. Elements in $\mathcal{W}(k)$ will be simply denoted by $g$. By using Hurwitz schemes, one can naturally endow $\mathcal{W}(k)$ with a structure of a complex analytic manifold of dimension $4 k-4$ (the symmetric polynomials on the $2 k-4$ branch values of each $g \in \mathcal{W}(k)$ together with the $2 k$ points $p_{j} \in \mathbb{C}^{*}$ give a local chart for $\left.\mathcal{W}(k)\right)$. Given an element $g \in \mathcal{W}(k)$, we define the height differential $\phi$ associated to $g$ as the unique meromorphic 1-form on $\overline{\mathbb{C}}$ with polar divisor

$$
(\phi)_{\infty}=\prod_{j=1}^{2 k} p_{j}
$$


whose residues are given by (3.2). Let $\mathcal{M}(k)$ be the set of elements $g \in \mathcal{W}(k)$ such that $(g, \phi)$ is the Weierstrass pair of a complete immersed minimal surface in $\mathbb{R}^{3} / T$ with Scherk-type ends, or equivalently

$$
\begin{aligned}
\mathcal{M}(k) & =\left\{g \in \mathcal{W}(k) \mid(\phi)_{0}=(g)_{0}(g)_{\infty} \text { and }\left|g\left(p_{j}\right)\right|=1,1 \leq j \leq 2 k\right\} \\
& =\left\{g \in \mathcal{W}(k) \mid(\phi)_{0}=(g)_{0}(g)_{\infty} \text { and }\left|\operatorname{Res}_{p_{j}}(g \phi)\right|=1,1 \leq j \leq 2 k\right\} .
\end{aligned}
$$

(Here $(h)_{0},(h)_{\infty}$ denote the zero and polar divisors of a meromorphic function or differential on $\overline{\mathbb{C}}$.) We can see the set of marked embedded surfaces $\widetilde{\mathcal{S}}(k)$ as a subset of $\mathcal{M}(k)$. Note that $\widetilde{\mathcal{S}}(k)$ is not open in $\mathcal{M}(k)$ (because the directions of the ends are not fixed; compare with the situation in Meeks-Pérez-Ros [9] or Pérez-Rodríguez-Traizet [13]).

Remark 3.1. Essentially what we do in the paper is prove that the only elements of $\mathcal{M}(k)$ which give embedded surfaces are the Karcher saddle towers. We will do this using a rather elaborate machinery developed in previous papers 9 . As elements of $\mathcal{M}(k)$ are defined by simple algebraic equations, one may wonder if there is a purely algebraic proof (following, for example, the method in Wei 20]). So, let us explain why a purely computational proof cannot succeed.

We have used embeddedness to obtain algebraic restrictions on the Weierstrass data (such as (3.1) above). However, there are examples of singly periodic minimal surfaces with Scherk-type ends and genus zero which satisfy all conditions we have written so far and yet are not embedded. Here is one example:

$$
g=\frac{z^{4}-4 i z^{2}+3}{z^{4}+4 i z^{2}+3}, \quad \phi=\frac{\left(z^{4}-4 i z^{2}+3\right)\left(z^{4}+4 i z^{2}+3\right) i d z}{\left(z^{2}+3\right)\left(z^{2}-3\right)\left(z^{2}+1\right)\left(z^{2}-1\right) z} .
$$

This example has 10 ends. The value of the complex Gauss map at the ends $0, \infty$ is 1 , while the ends at $\pm 1, \pm i, \pm \sqrt{3}, \pm i \sqrt{3}$ have Gauss map $\pm i$. This is an example of a special surface, and it is not embedded. Hence embeddedness must be used in a rather strong way, which is hard to perform in a purely computational proof.

Definition 3.2. The flux map $F: \mathcal{W}(k) \rightarrow \mathbb{C}^{2 k}$ is given by

$$
F(g)=\left(\operatorname{Res}_{p_{1}}(g \phi), \ldots, \operatorname{Res}_{p_{2 k}}(g \phi)\right),
$$

which is clearly holomorphic. Given $\mathbf{u} \in \mathcal{U}(k)$, let $\mathcal{M}_{\mathbf{u}}(k)=F^{-1}(\mathbf{u}) \cap \mathcal{M}(k)$ and $\widetilde{\mathcal{S}}_{\mathbf{u}}(k)=F^{-1}(\mathbf{u}) \cap \widetilde{\mathcal{S}}(k)$ (note that $F^{-1}(\mathbf{u})$ is not necessarily contained in $\mathcal{M}(k)$, since $\phi$ might not satisfy the regularity condition of the induced metric: $\left.(\phi)_{0}=(g)_{0}(g)_{\infty}\right)$.

Lemma 3.3. Given $\mathbf{u} \in \mathcal{U}(k)$, the subset $\widetilde{\mathcal{S}}_{\mathbf{u}}(k)$ is open and closed in $\mathcal{M}_{\mathbf{u}}(k)$.

Proof. Closeness follows since a limit of embedded surfaces is itself embedded. Any embedded surface in $\widetilde{\mathcal{S}}_{\mathbf{u}}(k)$ admits a regular neighborhood of constant positive radius (this is a consequence of the maximum principle if $\mathbf{u}$ is in the interior of $\mathcal{U}(k)$, and of the maximum principle at infinity if $\mathbf{u} \in \partial \mathcal{U}(k)$; see Ros [17 for a similar argument). From here the desired openness is standard.

We will later need the following property.

Lemma 3.4. Compact analytic subvarieties of $\mathcal{W}(k)$ are finite sets.

Proof. (Inspired in Meeks-Pérez-Ros 9.) We may assume after composition with Möbius transformations that for any element $g \in \mathcal{W}(k)$ we have $p_{1}=\infty, p_{2}=$ $0, p_{3}=1$. Then any element in $\mathcal{W}(k)$ has $k-1$ zeros and $k-1$ poles in $\mathbb{C}^{*}-\{1\}$. 
Consider the elementary symmetric functions $\sigma_{1}, \ldots, \sigma_{k-1}$ of the zeros, which are holomorphic functions on $\mathcal{W}(k)$. Let $A$ be a compact analytic subvariety of $\mathcal{W}(k)$. As $\sigma_{j}(A)$ is a compact analytic subvariety of $\mathbb{C}, \sigma_{j}(A)$ is finite. Since this works for any $j$, we deduce that there are only a finite number of possibilities in $\mathbb{C}^{*}-\{1\}$ for zeros of elements of $A$. In the same way, there are only a finite number of possibilities for poles of elements of $A$. Finally, the function $g \in \mathcal{W}(k) \mapsto g\left(p_{1}\right)$ is holomorphic, so again there are only a finite number of possibilities for $g\left(p_{1}\right)$, $g \in A$. As any $g \in \mathcal{W}$ is locally determined by its zeros, poles and its value in $\mathbb{C}^{*}$ at one point, this proves that $A$ is discrete. As $A$ is compact, it must be finite.

3.1. The ligature map. The ligature map $L$ will be a holomorphic mapping from $\mathcal{W}(k)$ to $\mathbb{C}^{4 k-4}$ that expresses when an element $g \in \mathcal{W}(k)$ lies in $\mathcal{M}(k)$. To define $L$ we cannot simply consider the value of $\phi / d z$ at the zeros and poles of $g$, because $g$ might have multiple zeros and in this case these zeros do not depend analytically on $g$. We may again assume by normalization that $p_{1}=\infty, p_{2}=0$ and $p_{3}=1$, and consequently write

$$
g=\lambda \frac{P_{1}(z)}{P_{2}(z)}, \quad \phi=i \frac{P_{3}(z)}{\prod_{j \geq 2}\left(z-p_{j}\right)} d z
$$

where $\lambda \in \mathbb{C}^{*}$, and $P_{1}, P_{2}, P_{3}$ are unitary polynomials of respective degrees $k-1$, $k-1$ and $2 k-2$ (this last degree comes from the fact that $\phi$ must have a simple pole at $\infty$; the factor $i$ in front of $\frac{P_{3}}{\prod_{j>2}\left(z-p_{j}\right)} d z$ comes from the equation $\operatorname{Res} \infty_{\infty} \phi=-i$, which follows from (3.2)). Note that the polynomials $P_{1}, P_{2}, P_{3}$ depend analytically on $g$. On the other hand, the regularity condition $(\phi)_{0}=(g)_{0}(g)_{\infty}$ is equivalent to $P_{1} P_{2}=P_{3}$, or also to

$$
\text { Remainder }\left(\frac{P_{3}}{P_{1} P_{2}}\right)=0 \text {. }
$$

As the above remainder of the euclidean division of $P_{3}$ by $P_{1} P_{2}$ is a complex polynomial of degree less than $2 k-2$, we can see it as a tuple in $\mathbb{C}^{2 k-2}$ by considering its coefficients.

Definition 3.5. The ligature map $L: \mathcal{W}(k) \rightarrow \mathbb{C}^{4 k-4}$ is the holomorphic map

$$
g \in \mathcal{W}(k) \mapsto L(g)=\left(\operatorname{Remainder}\left(P_{3} /\left(P_{1} P_{2}\right)\right), \operatorname{Res}_{p_{3}}(g \phi), \ldots, \operatorname{Res}_{p_{2 k}}(g \phi)\right) .
$$

We only consider the above residues for $3 \leq j \leq 2 k$, since the equations to solve (expressing when $g \in \mathcal{W}(k)$ lies in $\mathcal{M}(k))$ are not independent. Indeed, by the residue theorem

$$
\begin{gathered}
\sum_{\text {zeros of } g} \operatorname{Res}\left(g^{-1} \phi\right)+\sum_{j=1}^{2 k} \operatorname{Res}_{p_{j}}\left(g^{-1} \phi\right)=0, \\
\sum_{\text {poles of } g} \operatorname{Res}(g \phi)+\sum_{j=1}^{2 k} \operatorname{Res}_{p_{j}}(g \phi)=0 .
\end{gathered}
$$

Also observe that

$$
\operatorname{Res}_{p_{j}}\left(g^{-1} \phi\right)=\frac{(-1)^{j} i}{g\left(p_{j}\right)}=\frac{-1}{\operatorname{Res}_{p_{j}} g \phi} .
$$

Thus if $L(g)=\left(0,{ }^{(2 k-2)}, 0, u_{3}, \ldots, u_{2 k}\right)$ with $\mathbf{u}=\left(u_{1}, \ldots, u_{k}\right) \in \mathcal{U}(k)$, then we obtain a system of two equations for ( $\operatorname{Res}_{p_{1}}(g \phi)$, Res $\left.p_{2}(g \phi)\right)$, whose two solutions are $\left(u_{1}, u_{2}\right)$ and $\left(u_{2}, u_{1}\right)$. Hence $\mathcal{M}_{\mathbf{u}}(k)$ is a union of components of the subset of 
$\mathcal{W}(k)$ defined by $L(g)=\left(0, u_{3}, \ldots, u_{2 k}\right)$. Since $L$ is holomorphic, we deduce the following lemma.

Lemma 3.6. Given $\mathbf{u} \in \mathcal{U}(k), \mathcal{M}_{\mathbf{u}}(k)$ is a complex analytic subvariety of $\mathcal{W}(k)$.

\section{Proof of Theorem 2.6}

The proof of Theorem 2.6 is by induction on $k \geq 3$. Suppose that the theorem holds for any $k^{\prime}<k$, and we will prove it for $k$ (note that the theorem holds for $k=2$ ). We will assume the following three propositions, to be proven in further sections. Recall that $\widetilde{\mathcal{S}}_{0}(k)=F^{-1}\left(\mathcal{U}_{0}(k)\right)$ is the set of special surfaces with $2 k$ ends.

Proposition 4.1 (Properness).

(i) The flux map $F: \widetilde{\mathcal{S}}(k) \backslash \widetilde{\mathcal{S}}_{0}(k) \rightarrow \mathcal{U}(k) \backslash \mathcal{U}_{0}(k)$ is proper.

(ii) The flux map $F: \widetilde{\mathcal{S}}_{0}(k) \rightarrow \mathcal{U}_{0}(k)$ is proper.

Proposition 4.2 (Openness). The flux map $F: \widetilde{\mathcal{S}}(k) \rightarrow \mathcal{U}(k)$ is open.

Proposition 4.3 (Local uniqueness). There exists a point $\mathbf{u}_{*} \in \mathcal{U}_{0}(k)$ and $\varepsilon>0$ such that if $\mathbf{u} \in \mathcal{U}(k)$ satisfies $\left\|\mathbf{u}-\mathbf{u}_{*}\right\|<\varepsilon$, then $F^{-1}(\mathbf{u}) \subset \widetilde{\mathcal{K}}(k)$.

Assuming these results, we now prove Theorem 2.6. We first check that $\widetilde{\mathcal{S}}_{0}(k)$ is empty. By Proposition 4.2 and an elementary topological argument, the flux map $F: \widetilde{\mathcal{S}}_{0}(k) \rightarrow \mathcal{U}_{0}(k)$ is open. The same map is proper by point (ii) of Proposition 4.1. Hence the image by $F$ of any component of $\widetilde{\mathcal{S}}_{0}(k)$ is an entire component of $\mathcal{U}_{0}(k)$. Note that $\mathcal{U}_{0}(k)$ is not connected, but in any component of $\mathcal{U}_{0}(k)$ there is a degenerate polygon which reduces to a segment. By the maximum principle (see Remark 2.4), such a polygon cannot lie in the image of $F$. Therefore $\widetilde{\mathcal{S}}_{0}(k)=\varnothing$.

Then by Proposition 4.2 and elementary topology, the flux map $F: \widetilde{\mathcal{S}}(k) \rightarrow$ $\mathcal{U}(k) \backslash \mathcal{U}_{0}(k)$ is open. By point (i) of Proposition 4.1, it is also proper. Hence the image by $F$ of any component of $\widetilde{\mathcal{S}}(k)$ is all of $\mathcal{U}(k) \backslash \mathcal{U}_{0}(k)$ (which is connected). By Proposition 4.3 any component of $\widetilde{\mathcal{S}}(k)$ must contain some Karcher saddle towers. Since the set of saddle towers $\widetilde{\mathcal{K}}(k)$ forms a component of $\widetilde{\mathcal{S}}(k)$, we deduce that $\widetilde{\mathcal{S}}(k)=\widetilde{\mathcal{K}}(k)$. Now the remaining assertions in the statement of Theorem 2.6 follow easily.

Remark 4.4. Although we did not use the hypothesis of induction in the last paragraph, the proof of Proposition 4.1 needs Theorem 2.6 to be true for any $k^{\prime}<k$.

\section{Properness}

Along this section, $\left\{M_{n}\right\}_{n} \subset \widetilde{\mathcal{S}}(k)$ will denote a sequence of marked surfaces whose associated flux polygons $\mathbf{u}_{n}=F\left(M_{n}\right)$ converge as $n \rightarrow \infty$ to a polygon $\mathbf{u}_{\infty} \in \mathcal{U}(k)$. The goal of this section is to understand the limit of (a subsequence of) $\left\{M_{n}\right\}_{n}$.

5.1. Preliminaries on convex polygons. We need an elementary fact about convex unitary polygons. Recall that to each element $\mathbf{u}=\left(u_{1}, u_{2}, \ldots, u_{2 k}\right) \in \mathcal{U}(k)$ we associate a convex polygon $P_{\mathbf{u}}$ with vertices $Z_{1}, \ldots, Z_{2 k}$ such that for each $j=1, \ldots, 2 k, u_{j}=Z_{j}-Z_{j-1}$ (here we set $Z_{0}=Z_{2 k}$ ). Let $\alpha_{j}$ be the angle between $u_{j}$ and $u_{j+1}$. Thus $\beta_{j}=\pi-\alpha_{j}$ is the inner angle of the polygon at the vertex $Z_{j}$. 


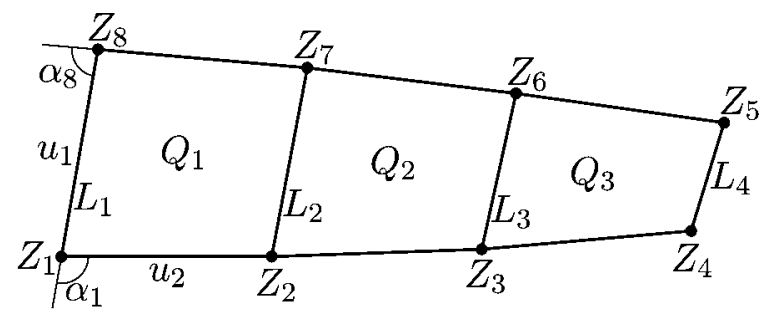

Figure 1. Definition of $\alpha_{j}, L_{j}$ and $Q_{j}(k=4)$.

Lemma 5.1. Given $\mathbf{u} \in \mathcal{U}(k)$ and $i=1, \ldots, 2 k$, it holds that $\alpha_{i}+\alpha_{i+1} \leq \pi$, with equality for some $i$ if and only if $\mathbf{u} \in \mathcal{U}_{0}(k)$.

Proof. To simplify notation, we do not lose generality assuming that $i=2 k$ in the proposition. Then for $j=1, \ldots, k$ we consider the length $L_{j}=Z_{j} Z_{2 k-j+1}=$ $\left|\overline{Z_{j} Z_{2 k-j+1}}\right|$, and for $j=1, \ldots, k-1$ the quadrilaterals $Q_{j}$ with vertices $Z_{j}, Z_{j+1}$, $Z_{2 k-j}, Z_{2 k-j+1}$; see Figure1. Since $P_{\mathbf{u}}$ is convex, each $Q_{j}$ is a convex quadrilateral.

Now assume that $\alpha_{2 k}+\alpha_{1} \geq \pi$. We want to prove that $\mathbf{u}$ is special. By convexity of $P_{\mathbf{u}}$ and since $\beta_{2 k}+\beta_{1} \leq \pi$, for each $j=1, \ldots, k-1$ the quadrilateral $Q_{j}$ has the following property: the sum of the inner angles of $Q_{j}$ at the two endpoints corresponding to $L_{j}$ (do not confuse these inner angles with those of $P_{\mathbf{u}}$, which are $\beta_{j}$ and $\beta_{2 k-j+1}$ ) is not greater than $\pi$. By Lemma 5.2 below, we have $L_{j+1} \leq L_{j}$, with equality if and only if $Q_{j}$ is a parallelogram. This gives $L_{1} \geq L_{2} \geq \ldots \geq L_{k}$. But $L_{1}=L_{k}=1$ because these are edges of the polygon $P_{\mathbf{u}}$. Hence equality holds everywhere, which implies that all $Q_{j}$ are parallelograms, so $\mathbf{u}$ is special.

Lemma 5.2. Let $A B C D$ be a convex quadrilateral. Let $\widehat{A}$ and $\widehat{B}$ be the inner angles at $A$ and $B$. Assume that $B C=A D$ and $\widehat{A}+\widehat{B} \leq \pi$. Then $D C \leq A B$, with equality if and only if $A B C D$ is a parallelogram.

Proof. Without loss of generality we may assume that $B C=A D=1$. Let us fix the points $A, B, D$ and see how the length $l(t)=D C_{t}=\left|\overline{D C_{t}}\right|$ varies as a function of $\widehat{B}=t$. Then

$$
\frac{1}{2} \frac{d}{d t}\left(l(t)^{2}\right)=\left\langle\overrightarrow{D C}_{t}, \frac{d C_{t}}{d t}\right\rangle=\left\langle\overrightarrow{D C}_{t}, \vec{u}_{t}\right\rangle
$$

where $\vec{u}_{t}$ is a unitary vector perpendicular to $\overrightarrow{B C}_{t}$. But provided the quadrilateral is convex, this scalar product is positive, so $l(t)$ is an increasing function of $t=\widehat{B}$ (in fact $l(t)$ is minimum precisely when $B, C_{t}, D$ are on a line). When $t=\pi-\widehat{A}$, the quadrilateral $A B C_{t} D$ is a parallelogram, in which case $l(\pi-\widehat{A})=A B$. Thus $l(t) \leq l(\pi-\widehat{A})$ whenever $t \leq \pi-\widehat{A}$, which proves the lemma.

Remark 5.3. Clearly Lemma 5.1 does not hold for convex unitary polygons with an odd number of vertices.

5.2. Area, flux and curvature estimates. As usual, we denote by $\widetilde{M}$ the lift to $\mathbb{R}^{3}$ of a surface $M \subset \mathbb{R}^{3} / T$, by $B(x, R)$ the open ball centered at $x \in \mathbb{R}^{3}$ with radius $R>0$, by $C(R) \subset \mathbb{R}^{3} / T$ the solid vertical cylinder of radius $R$ and axis $\mathbb{R} T$ modded out by $T$, and by $K_{\Sigma}$ the Gauss curvature function of a surface $\Sigma \subset \mathbb{R}^{3}$. 
Lemma 5.4. Given $M \in \mathcal{M}(k)$, the area of $\widetilde{M} \cap B(R)$ is less than $k \pi R^{2}$ for any $R>0$.

Proof. Since $\widetilde{M}$ is asymptotic to $2 k$ vertical halfplanes, the limit of $\frac{\operatorname{Area}(\widetilde{M} \cap B(R))}{R^{2}}$ when $R \rightarrow \infty$ is $k \pi$. Now the result follows from the monotonicity formula.

The fact that the genus of our surfaces is zero allows us to control the flux of vertical sections of our sequence $\left\{M_{n}\right\}_{n} \subset \widetilde{\mathcal{S}}(k)$ with $\left\{\mathbf{u}_{n}=F\left(M_{n}\right)\right\}_{n} \rightarrow \mathbf{u}_{\infty} \in$ $\mathcal{U}(k)$.

Lemma 5.5. There exist $a \in \mathbb{S}^{1}$ and $\varepsilon>0$ depending only on $\mathbf{u}_{\infty}$, such that for any flat vertical annulus $\Pi \subset \mathbb{R}^{3} / T, n \in \mathbb{N}$ and any component $\gamma_{n}$ of $M_{n} \cap \Pi$,

$$
\left|\operatorname{Flux}\left(\gamma_{n}\right) \pm 2 \pi a\right| \geq \varepsilon
$$

Proof. Fix a flat vertical annulus $\Pi \subset \mathbb{R}^{3} / T$ and a component $\gamma_{n}$ of $M_{n} \cap \Pi$. Since $M_{n}$ has genus zero, $\gamma_{n}$ separates $M_{n}$ in two components. Hence the flux of $M_{n}$ along $\gamma_{n}$ is equal to the sum of the fluxes at some of the ends of $M_{n}$. In particular, $\operatorname{Flux}\left(\gamma_{n}\right)$ is horizontal (we will see it in $\mathbb{C}$ ) and for $n$ fixed there are only a finite number of values in $\mathbb{C}$ for $\operatorname{Flux}\left(\gamma_{n}\right)$, that only depend on $\mathbf{u}_{n}$. Now the existence of $a \in \mathbb{S}^{1}$ and $\varepsilon>0$ verifying (5.1) follows from the fact that $\left\{\mathbf{u}_{n}\right\}_{n}$ converges to $\mathbf{u}_{\infty}$ as $n \rightarrow \infty$.

Let $g_{n}$ be the Gauss map of $M_{n}$ (stereographically projected from the north pole). A direct consequence of Lemma 5.5 is that for all $n$ large, none of the ends of $M_{n}$ has limit normal vector $i a$.

Lemma 5.6. Let $a \in \mathbb{S}^{1}$ be the unit complex number given by Lemma 5.5. Then, there exists $\delta>0$ depending only on $\mathbf{u}_{\infty}$ such that for all $n \in \mathbb{N}$ and $p_{n} \in M_{n}$ such that $g_{n}\left(p_{n}\right)=i a$,

$$
\sup \left|K_{\left(M_{n}-p_{n}\right) \cap C(10)}\right| \geq \delta .
$$

Proof. By contradiction, if $\sup \left|K_{\left(M_{n}-p_{n}\right) \cap C(10)}\right|$ converges to zero as $n \rightarrow \infty$, then $\left\{\left(M_{n}-p_{n}\right) \cap C(10)\right\}_{n}$ converges uniformly to the flat vertical annulus $\Pi_{1}$ with normal $i a$. Now take a flat vertical annulus $\Pi$ perpendicular to $\Pi_{1}$. Then, the flux of $M_{n}$ along $M_{n} \cap \Pi=\gamma_{n}$ converges to $\pm 2 \pi a$ as $n \rightarrow \infty$, which contradicts Lemma 5.5

5.3. Weak limits. We now prove that $\left\{M_{n}\right\}_{n}$ converges in some weak sense to a finite number of limit minimal surfaces. This ought to be true in a quite general setup, although we will give a proof enough for our setting.

Let $a \in \mathbb{S}^{1}$ be given by Lemma 5.5. For each $n \in \mathbb{N}$ let $p_{1, n}, \ldots, p_{k-1, n} \in M_{n}$ be the points such that $g_{n}\left(p_{j, n}\right)=i a$ for $1 \leq j \leq k-1$ (counting with multiplicity). Let $\widetilde{p}_{1, n}, \ldots, \widetilde{p}_{k-1, n} \in \widetilde{M}_{n}$ be their corresponding liftings to a fundamental domain of $\widetilde{M}_{n}$. Fix $j=1, \ldots, k-1$. Since the degree of $g_{n}$ is fixed $k-1$, given $R>0$ there exists $c_{1}>0$ such that the absolute total curvature of $\left(\widetilde{M}_{n}-\widetilde{p}_{j, n}\right) \cap B(R)$ is not greater than $c_{1}$ for all $n \in \mathbb{N}$. Furthermore, Lemma 5.4 implies that there exists $c_{2}>0$ such that Area $\left(\left(\widetilde{M}_{n}-\widetilde{p}_{j, n}\right) \cap B(R)\right) \leq c_{2}$ for all $n \in \mathbb{N}$. In this situation, a standard result (see e.g. Theorem 4.40 in [16]) insures that there exists a discrete set $X_{j} \subset \mathbb{R}^{3}$ and a properly embedded minimal surface $\widetilde{M}_{j, \infty} \subset \mathbb{R}^{3}$ such that up to 
a subsequence, $\left\{\widetilde{M}_{n}-\widetilde{p}_{j, n}\right\}_{n}$ converges with finite multiplicity in $\mathbb{R}^{3}-X_{j}$ to $\widetilde{M}_{j, \infty}$. Furthermore, for each point $p \in X_{j}$ and $\mu>0$, it holds that

$$
\underset{k}{\limsup } \int_{\left(\widetilde{M}_{n}-\widetilde{p}_{j, n}\right) \cap B(p, \mu)}\left|K_{n}\right| \geq 4 \pi .
$$

We now distinguish two possibilities, depending on whether $\widetilde{M}_{j, \infty}$ is flat or not:

I. If $\widetilde{M}_{j, \infty}$ is not flat, then by a standard argument (see e.g. Section 4 of [16]) the multiplicity of the convergence of $\left\{\widetilde{M}_{n}-\widetilde{p}_{j, n}\right\}_{n}$ to $\widetilde{M}_{j, \infty}$ is one and $X_{j}=\varnothing$. Since each $\widetilde{M}_{j, n}$ is $T$-periodic, the same holds for $\widetilde{M}_{j, \infty}$. The quotient surface $M_{j, \infty}=\widetilde{M}_{j, \infty} / T$ is a planar domain since all the $M_{n}$ have genus zero, and its absolute total curvature is at most $4 \pi(k-1)$. Therefore all ends of $M_{j, \infty}$ are simultaneously planar, helicoidal or Scherk-type by a theorem of Meeks and Rosenberg [11]. As $M_{j, \infty}$ is an embedded nonflat planar domain, the maximum principle implies that $M_{j, \infty}$ does have planar ends. If the ends of $M_{j, \infty}$ are helicoidal, then it is a helicoid (Pérez and Ros [14]). As $T$ is vertical, such a helicoid must also be vertical. Otherwise, $M_{j, \infty}$ has genus zero and at most $2 k$ Scherk-type ends.

II. If $\widetilde{M}_{j, \infty}$ is flat, then $X_{j} \neq \varnothing$ by Lemma [5.6] The proof of Theorem 4.40 in [16] implies that there exists a sequence of real numbers $\lambda_{n} \rightarrow+\infty$ such that $\lambda_{n}\left(\widetilde{M}_{n}-\widetilde{p}_{j, n}\right)$ converges to a properly embedded nonflat minimal surface $\widehat{M}_{j, \infty}$. Since the $M_{n}$ are planar domains, $\widehat{M}_{j, \infty}$ must have genus zero and so, it is a catenoid (López and Ros [7]). As the neck of $\widehat{M}_{j, \infty}$ is a closed curve with nonzero period, it must be the uniform limit of closed curves with nonzero flux in the $M_{n}$ (rescaled by $\lambda_{n}$ ). As all of such curves on $M_{n}$ have horizontal flux, $\widehat{M}_{j, \infty}$ is a horizontal catenoid.

Lemma 5.7. In the situation above, suppose that $M_{j, \infty}$ is a limit surface of the type I. Then, none of the ends of $M_{j, \infty}$ has limit normal vector $i a$.

Proof. If the ends of $M_{j, \infty}$ are helicoidal, then the lemma is trivial. Arguing by contradiction, suppose that $M_{j, \infty}$ has Scherk-type ends (which must be vertical because $\widetilde{M}_{j, \infty}$ is $T$-invariant), one of which has limit normal vector $i a$. Thus we can find a flat vertical annulus $\Pi \subset \mathbb{R}^{3} / T$ orthogonal to $a$ such that the flux of $M_{j, \infty}$ along a certain component $\gamma_{j, \infty}$ of $M_{j, \infty} \cap \Pi$ is $\pm 2 \pi a . \gamma_{j, \infty}$ is the uniform limit of components $\gamma_{n}$ of $M_{n} \cap \Pi$, which therefore have flux converging to $\pm 2 \pi a$, a contradiction with Lemma 5.5 .

We now need to get rid of duplicate limits.

Lemma 5.8. In the situation of Lemma 5.7, let $d_{j}$ be the degree of the Gauss map on $M_{j, \infty}$ (thus $\left.d_{j} \leq k-1\right)$. Then, there are another $d_{j}-1$ points $q_{1, n}(j), \ldots, q_{d_{j}-1}(j)$ $\in\left\{\widetilde{p}_{1, n}, \ldots, \widetilde{p}_{k-1, n}\right\} \backslash\left\{\widetilde{p}_{j, n}\right\}$ such that for every $h=1 \ldots, d_{j}-1$, a translation of $\widetilde{M}_{j, \infty}$ is also the limit of $\left\{\widetilde{M}_{n}-q_{h, n}(j)\right\}_{n}$ as $n \rightarrow \infty$.

Proof. By Lemma [5.7, there exists a compact region $C \subset \mathbb{R}^{3} / T$ such that for $n$ large, $g_{n}$ takes the value $i a$ at $d_{j}$ points in $C$ counting multiplicity (one of these points is the origin). The remaining $d_{i}-1$ points correspond to other points $q_{1, n}, \ldots, q_{d_{j-1}} \in\left\{\widetilde{p}_{1, n}, \ldots, \widetilde{p}_{k-1, n}\right\} \backslash\left\{\widetilde{p}_{j, n}\right\}$, and clearly $\left\{\widetilde{M}_{n}-q_{h, n}\right\}_{n}$ converges to $\widetilde{M}_{j, \infty}$ (up to a translation) as $n \rightarrow \infty$. 
For the remainder of this section, we forget about the repeated limits as $n \rightarrow \infty$ of the $M_{n}-q_{h, n}(j)$ in Lemma [5.8, since they are the same as $M_{j, \infty}$. Let $r$ be the number of limit surfaces that remain. After re-indexing, we obtain that for each $j=1, \ldots, r$ the sequence $\left\{M_{n}-p_{j, n}\right\}_{n}$ converges (up to possibly blowing-up) to $M_{j, \infty}$ which is either singly periodic or a horizontal catenoid. By counting the number of times where the Gauss map takes the value $i a$ we deduce that the degrees of the Gauss maps of all the $M_{j, \infty}$ add up to $k-1$. Furthermore, there exist $r$ disjoint metric balls $B_{j, n}$ in $\mathbb{R}^{3} / T$ such that $M_{n} \cap B_{j, n}$ traps all the interesting geometry of the limit $M_{j, \infty}$, and the total curvature of $M_{n} \cap B_{j, n}$ is arbitrarily close to the total curvature of $M_{j, \infty}$. In particular, the total curvature of $M_{n} \backslash\left(B_{1, n} \cup \ldots \cup B_{r, n}\right)$ is arbitrarily small.

Note that for $j=1, \ldots, r$ fixed, the Gauss map at each end representative of $M_{j, \infty}$ is close to a constant that depends on the end. For $n$ large, let $\Omega$ be a component of $M_{n} \backslash\left(B_{1, n} \cup \ldots \cup B_{r, n}\right)$. By the open mapping theorem, the Gauss map $g_{n}$ of $M_{n}$ has to be close to a constant $c(\Omega)$ on $\Omega$ (otherwise $g_{n}(\Omega)$ would cover almost all the sphere). Hence $\Omega$ is an extremely flat graph over a certain plane (quotiented by $T$ ). Now let $\Omega$ be a noncompact component of $M_{n} \backslash\left(B_{1, n} \cup \ldots \cup B_{r, n}\right)$. Since the ends of $M_{n}$ have a horizontal limit normal vector, we have $|c(\Omega)|=1$. As the Gauss map of a helicoid in $\mathbb{R}^{3} / T$ is vertical at its ends, we deduce that for any ball $B_{j_{0}, n}$ such that $\partial B_{j_{0}, n} \cap \partial \Omega \neq \varnothing$, the associated limit surface $M_{j_{0}, \infty}$ is either a horizontal catenoid or a properly embedded planar domain with vertical Scherk-type ends. Therefore for any component $\Omega^{\prime}$ of $M_{n} \backslash\left(B_{1, n} \cup \ldots \cup B_{r, n}\right)$ with $\partial B_{j_{0}, n} \cap \partial \Omega^{\prime} \neq \varnothing$, it holds that $\left|c\left(\Omega^{\prime}\right)\right|=1$. We now repeat inductively this process exchanging $\Omega$ by $\Omega^{\prime}$, to finally conclude that all the limit surfaces $M_{j, \infty}$ are horizontal catenoids or properly embedded minimal planar domains with vertical Scherk-type ends.

We now summarize what we have proven in the following statement.

Proposition 5.9. There exists a collection $M_{1, \infty}, \ldots, M_{r, \infty}$ of minimal surfaces and $r$ sequences of homotheties $\left\{h_{j, n}\right\}_{n}$ satisfying

(1) Each $M_{j, \infty}$ is a horizontal catenoid or a properly embedded minimal surface of genus zero in $\mathbb{R}^{3} / T$ with at most $2 k$ Scherk-type ends.

(2) $\int_{M_{n}}\left|K_{M_{n}}\right|=\sum_{j=1}^{n} \int_{M_{j, \infty}}\left|K_{M_{j, \infty}}\right|$, for each $n \in \mathbb{N}$.

(3) If $M_{j, \infty}$ is a catenoid, then the sequence of scaling factors of $h_{n}$ blows-up and $\left\{h_{j, n}\left(M_{n}\right)\right\}_{n}$ converges smoothly to $M_{j, \infty}$ in $\mathbb{R}^{3}$.

(4) If $M_{j, \infty}$ has Scherk-type ends, then each $h_{n}$ is a translation and $\left\{h_{j, n}\left(M_{n}\right)\right\}_{n}$ converges smoothly to $M_{j, \infty}$ in $\mathbb{R}^{3} / T$.

(5) For $R, n$ large, there exist $r$ disjoint metric balls $B_{j, n} \subset \mathbb{R}^{3} / T$ such that $h_{j, n}\left(B_{j, n}\right)$ is either the ball $B(0, R) \subset \mathbb{R}^{3}$ (when $M_{j, \infty}$ is a catenoid) or the metric ball of radius $R$ centered at the origin in $\mathbb{R}^{3} / T$ (when $M_{j, \infty}$ has Scherk-type ends), and $M_{n}$ decomposes as

$$
M_{n}=\left(M_{n} \cap B_{1, n}\right) \cup \ldots \cup\left(M_{n} \cap B_{r, n}\right) \cup \Omega_{1, n} \cup \ldots \cup \Omega_{m, n} .
$$

Furthermore, each $\Omega_{h, n}$ is a graph over a domain in a flat vertical annulus $\Pi \subset \mathbb{R}^{3} / T$.

In the sequel, we will use the term connection piece to refer to each of the domains $\Omega_{h, n}$. Note that a connection piece could be compact (if it does not contains ends of $M_{n}$ ); see Figure 2 left. 

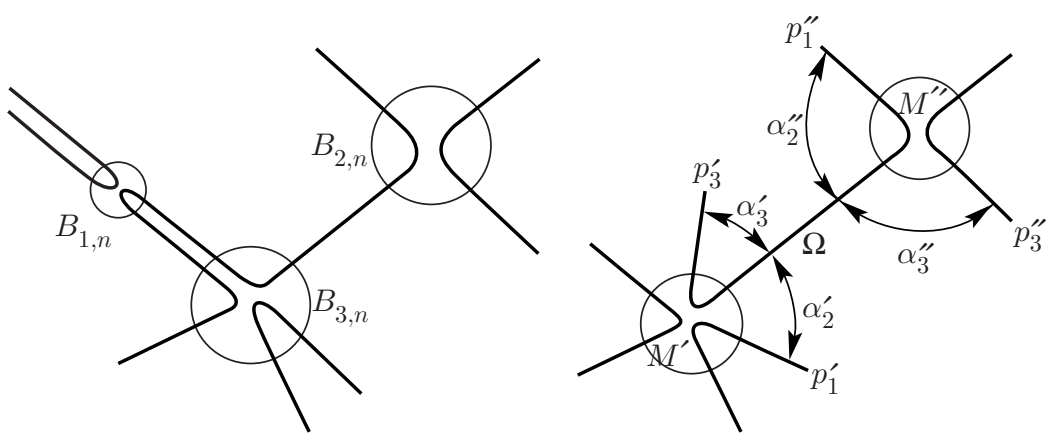

Figure 2. Left: A weak limit configuration with 3 limit surfaces ( $M_{1, \infty}$ is a catenoid, $M_{2, \infty}$ a Scherk surface and $M_{3, \infty}$ a surface of genus zero with six Scherk-type ends) and 11 connection pieces (3 of them being compact). Right: See Lemma 5.10.

5.4. Strong compactness. In this section we shall prove that if $\mathbf{u}_{\infty} \in \mathcal{U}(k) \backslash \mathcal{U}_{0}(k)$, then the collection of limit surfaces in Proposition 5.9 reduces to a single surface in $\widetilde{\mathcal{S}}(k)$ (this is usually referred to in the literature as strong compactness of the original sequence $\left\{M_{n}\right\}_{n} \subset \widetilde{\mathcal{S}}(k)$ ), and obtain further information for the possibly noncompact case, i.e. $\mathbf{u}_{\infty} \in \mathcal{U}_{0}(k)$.

Assume that $r \geq 2$ (we use the notation in Proposition 5.9). Consider two limit surfaces $M^{\prime}=M_{j_{1}, \infty}$ and $M^{\prime \prime}=M_{j_{2}, \infty}$ which are attached in $M_{n}$ by a compact connection piece $\Omega$. We label the ends of $M^{\prime}$ as $p_{j}^{\prime}$ and those of $M^{\prime \prime}$ as $p_{j}^{\prime \prime}$, in such a way that the ends $p_{2}^{\prime}$ and $p_{2}^{\prime \prime}$ are attached along $\Omega$. Let $u_{j}^{\prime}$ and $u_{j}^{\prime \prime}$ be the corresponding flux vectors. As in Subsection 5.1, let $\alpha_{j}^{\prime}$ be the angle between $u_{j-1}^{\prime}$ and $u_{j}^{\prime}$, and define $\alpha_{j}^{\prime \prime}$ analogously with $u_{j-1}^{\prime \prime}, u_{j}^{\prime \prime}$; see Figure 2 right.

Lemma 5.10. In the above situation, $\alpha_{2}^{\prime}+\alpha_{3}^{\prime \prime} \geq \pi$ and $\alpha_{2}^{\prime \prime}+\alpha_{3}^{\prime} \geq \pi$.

Proof. By contradiction, suppose $\alpha_{2}^{\prime}+\alpha_{3}^{\prime \prime}<\pi$. If both connection pieces that glue to $p_{1}^{\prime}, p_{3}^{\prime \prime}$ are noncompact, then these connection pieces are graphs over flat vertical halfplanes (quotiented by $T$ ) hence they intersect, which contradicts that $M_{n}$ is embedded. Hence at least one of the connection pieces $\Omega^{\prime}$, say that which glues to $p_{1}^{\prime}$, is compact. So $p_{1}^{\prime}$ is attached along $\Omega^{\prime}$ to an end of another weak limit $M^{\prime \prime \prime}$, and we repeat the discussion with the same $p_{3}^{\prime \prime}$, exchanging $p_{1}^{\prime}$ by a suitable end of $M^{\prime \prime \prime}$. After a finite chain of compact connection pieces and weak limits, either $p_{1}^{\prime}$ and $p_{3}^{\prime \prime}$ glue to different ends of $M_{n}$, or they glue each other through an almost horizontal compact arc $\Gamma$ that only intersects the closure of $\Omega$ at the end points of $\Gamma$. In the first case we find a contradiction as above, while in the second case we join $\Gamma$ with a suitable almost horizontal arc $\Gamma^{\prime} \subset \Omega$, so that $\Gamma \cup \Gamma^{\prime}$ is a closed curve in $M_{n}$. Since the metric balls $B_{j, n}$ in Proposition 5.9 all have bounded radius and for $n$ sufficiently large the length of the parts of $\Gamma \cup \Gamma^{\prime}$ in the connection pieces is as large as we desire, we deduce that for $n$ large, the flux of $M_{n}$ along $\Gamma \cup \Gamma^{\prime}$ is not horizontal, which contradicts the fact that such a flux vector is a sum of fluxes at the ends of $M_{n}$. Thus $\alpha_{2}^{\prime}+\alpha_{3}^{\prime \prime} \geq \pi$, and similarly $\alpha_{2}^{\prime \prime}+\alpha_{3}^{\prime} \geq \pi$. 
We continue analyzing the case $r \geq 2$. With the notation above, suppose that $M^{\prime}, M^{\prime \prime}$ have Scherk-type ends. By Lemma 5.1 applied to the corresponding polygon fluxes $F\left(M^{\prime}\right), F\left(M^{\prime \prime}\right)$, it holds that $\alpha_{2}^{\prime}+\alpha_{3}^{\prime} \leq \pi$ and $\alpha_{2}^{\prime \prime}+\alpha_{3}^{\prime \prime} \leq \pi$. Adding these inequalities and using Lemma 5.10 we conclude that $\alpha_{2}^{\prime}+\alpha_{3}^{\prime}=\alpha_{2}^{\prime \prime}+\alpha_{3}^{\prime \prime}=\pi$, so Lemma 5.1 gives that $F\left(M^{\prime}\right) \in \mathcal{U}_{0}\left(k^{\prime}\right), F\left(M^{\prime \prime}\right) \in \mathcal{U}_{0}\left(k^{\prime \prime}\right)$, where $k^{\prime}, k^{\prime \prime}$ are half of the number of ends of $M^{\prime}, M^{\prime \prime}$ respectively. Furthermore, $F\left(M^{\prime}\right)$ and $F\left(M^{\prime \prime}\right)$ have parallel sides. Thus all the fluxes at ends of $M^{\prime}$ are $\pm 2 \pi u^{\prime}$ except two of them which are $\pm 2 \pi v$ (here $u, v$ are distinct points in $\mathbb{S}^{1}$ ), and similarly with $M^{\prime \prime}$. Note that the connection piece $\Omega$ between $M^{\prime}$ and $M^{\prime \prime}$ is attached to ends with flux $\pm 2 \pi v$ of $M^{\prime}, M^{\prime \prime}$ (otherwise we again produce a closed curve in $M_{n}$ with a nonhorizontal flux vector).

Another consequence of these arguments is that all remaining weak limits $M_{j, \infty}$ other that $M^{\prime}, M^{\prime \prime}$ have necessarily Scherk-type ends, so we can repeat the arguments in the last paragraph to obtain that $\mathbf{u}_{\infty}=\lim F\left(M_{n}\right)$ belongs to $\mathcal{U}_{0}(k)$. Now we can deduce the main result of this section. Recall that $\mathbf{u}_{0}(k)$ is the degenerated special polygon all of whose edges are parallel (namely, the segment with endpoints $0, k)$, and that $F^{-1}\left(\mathbf{u}_{0}(k)\right)=\varnothing$ by the maximum principle.

Proposition 5.11. Let $\left\{M_{n}\right\}_{n} \subset \widetilde{\mathcal{S}}(k)$ with $\left\{F\left(M_{n}\right)=\mathbf{u}_{n}\right\}_{n} \rightarrow \mathbf{u}_{\infty}$.

(A) If $\mathbf{u}_{\infty} \notin \mathcal{U}_{0}(k)$, then after passing to a subsequence, $\left\{M_{n}\right\}_{n}$ converges to a single marked surface $M_{\infty} \in \widetilde{\mathcal{S}}(k) \backslash \widetilde{\mathcal{S}}_{0}(k)$.

(B) If $\mathbf{u}_{\infty} \in \mathcal{U}_{0}(k) \backslash\left\{\mathbf{u}_{0}(k)\right\}$, then after passing to a subsequence either $\left\{M_{n}\right\}_{n}$ converges to a single marked surface $M_{\infty} \in \widetilde{\mathcal{S}}_{0}(k)$, or all weak limit surfaces $M_{j, \infty}$ are special surfaces with less than $2 k$ ends.

(C) If $\mathbf{u}_{\infty}=\mathbf{u}_{0}(k)$, then after passing to a subsequence $\left\{M_{n}\right\}_{n}$ converges weakly to $k-1$ horizontal catenoids.

We finish this section by proving Proposition 4.1, Item (i) of this proposition is precisely point (A) of Proposition [5.11] To prove item (ii), consider a sequence of special surfaces $\left\{M_{n}\right\}_{n} \subset \widetilde{\mathcal{S}}_{0}(k)$ with $\left\{F\left(M_{n}\right)=\mathbf{u}_{n}\right\}_{n} \rightarrow \mathbf{u}_{\infty} \in \mathcal{U}_{0}(k)$.

If $\mathbf{u}_{\infty} \in \mathcal{U}_{0}(k) \backslash\left\{\mathbf{u}_{0}(k)\right\}$, then Proposition [5.11(B) asserts that either the $M_{n}$ converge to a single marked surface $M_{\infty} \in \widetilde{\mathcal{S}}_{0}(k)$ (which is what we want to prove), or all weak limit surfaces $M_{1, \infty}, \ldots, M_{r, \infty}$ of the sequence $\left\{M_{n}\right\}_{n}$ are special surfaces with less than $2 k$ ends. By induction hypothesis, Theorem 2.6 holds for any $k^{\prime}<k$, so we have $\widetilde{\mathcal{S}}_{0}\left(k^{\prime}\right)=\varnothing$ for each $k^{\prime}<k$ unless $k^{\prime}=2$, hence $M_{1, \infty}, \ldots, M_{r, \infty}$ are singly periodic Scherk minimal surfaces. These weak limits are in fact the same Scherk surface, because $\mathbf{u}_{\infty} \in \mathcal{U}_{0}(k)$. We will call this limit configuration with $k-1$ copies of a singly periodic Scherk surface a Scherk limit (note there exists a 1-parameter family of Scherk limits). In Section 7 we will study each Scherk limit, proving in particular that a sequence of special surfaces cannot converge to a Scherk limit, so this case does not happen.

Finally assume that $\mathbf{u}_{\infty}=\mathbf{u}_{0}(k)$. By Proposition 5.11(C), after a blow-up $\left\{M_{n}\right\}_{n}$ converges to $k-1$ copies of the same horizontal catenoid, a configuration that we will call the catenoid limit. In Section 8 we will see that a sequence of special surfaces cannot converge to the catenoid limit, so this case is also impossible. This completes the proof of Proposition 4.1 


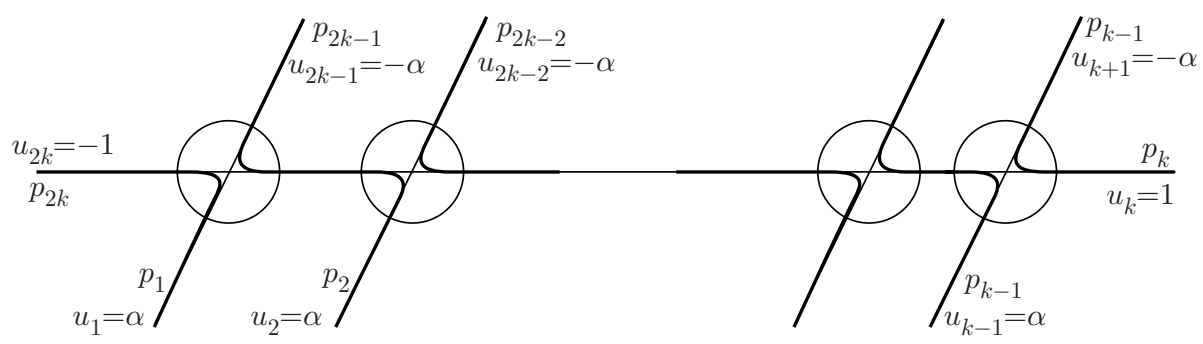

Figure 3. A Scherk limit configuration, with the ends and the corresponding fluxes $u_{j}$.

\section{OpenNESS}

In this section we will prove Proposition 4.2 Since openness is a local property, it suffices to see that given $M \in \widetilde{\mathcal{S}}(k)$, there exists an open neighborhood $V$ of $M$ in $\widetilde{\mathcal{S}}(k)$ such that $\left.F\right|_{V}: V \rightarrow \mathcal{U}(k)$ is an open map. Fix $M \in \widetilde{\mathcal{S}}(k)$. Consider the associated element $g$ in $\mathcal{W}(k)$ and flux polygon $\mathbf{u}=F(M) \in \mathcal{U}(k)$. Clearly $g \in \widetilde{\mathcal{S}}_{\mathbf{u}}(k)$. By Lemma 3.3 and Lemma 3.6. $\widetilde{\mathcal{S}}_{\mathbf{u}}(k)$ is an analytic subvariety of $\mathcal{W}(k)$. By the properness Proposition 4.1, $\widetilde{\mathcal{S}}_{\mathbf{u}}(k)$ is compact, hence it is finite by Lemma 3.4. Thus there exists an open set $\Omega \subset \mathcal{W}(k)$ such that $g \in \Omega$ and $L^{-1}(L(g))=\{g\}$. Since $L$ is holomorphic between complex manifolds of the same dimension, the open mapping theorem for finite maps (see [3], page 667) implies there exists an open set $\Omega_{1} \subset \Omega$ containing $g$ such that $\left.L\right|_{\Omega_{1}}$ is open. Now Proposition 4.2 follows directly using the relationship between $L$ and $F$.

\section{UNIQUENESS AROUND ANY SCHERK LIMIT}

In this section we will study surfaces close to the Scherk limits. We will conclude from this study two facts: First, that a sequence of special surfaces cannot converge to a Scherk limit, which was used in the proof of Proposition 4.1. Second, that all surfaces close to a Scherk limit must be Karcher saddle towers, which will be used to prove Proposition 4.3 .

Recall that a Scherk limit is a configuration of $k-1$ copies of the same singly periodic Scherk minimal surface. In Section 2 we normalized the space $\mathcal{U}(k)$ of convex unitary polygons to have their first component equal 1. In what follows we will slightly change this normalization, which does not affect the arguments but simplifies the notation. After rotation and suitable choice of the first end for $M_{n}$, we may assume that the limit of $\left\{F\left(M_{n}\right)\right\}_{n}$ is $\mathbf{u}_{\infty}=\left(\alpha,{ }^{(k-1)}, \alpha, 1,-\alpha,{ }^{(k-1)},-\alpha,-1\right)$, where $\alpha \in \mathbb{S}^{1}-\{ \pm 1\}$ and the ends $p_{1}, \ldots, p_{2 k}$ are labeled as indicated in Figure 3, A little thought of how the normal vector behaves when gluing consecutive copies of the Scherk surface shows that if we fix the normal map $g$ to be $i$ at the end $p_{2 k}$, then it alternates the values $\pm i$ in consecutive annular connection pieces between copies of the Scherk surface, finishing at $g\left(p_{k}\right)=(-1)^{k+1} i$.

7.1. Weierstrass data. A model for an element in $\mathcal{W}(k)$ close to the Scherk limit is as follows. Fix $\mathbf{u}=\left(u_{1}, \ldots, u_{2 k}\right) \in \mathbb{C}^{2 k}$ close to $\mathbf{u}_{\infty}$ (we do not require the components of $\mathbf{u}$ either to be unitary or to satisfy equation (2.3) ). Consider $k-1$ copies $\overline{\mathbb{C}}_{1}, \ldots, \overline{\mathbb{C}}_{k-1}$ of $\overline{\mathbb{C}}$. For $1<j<k$, consider complex numbers $a_{j} \neq b_{j}$ close 


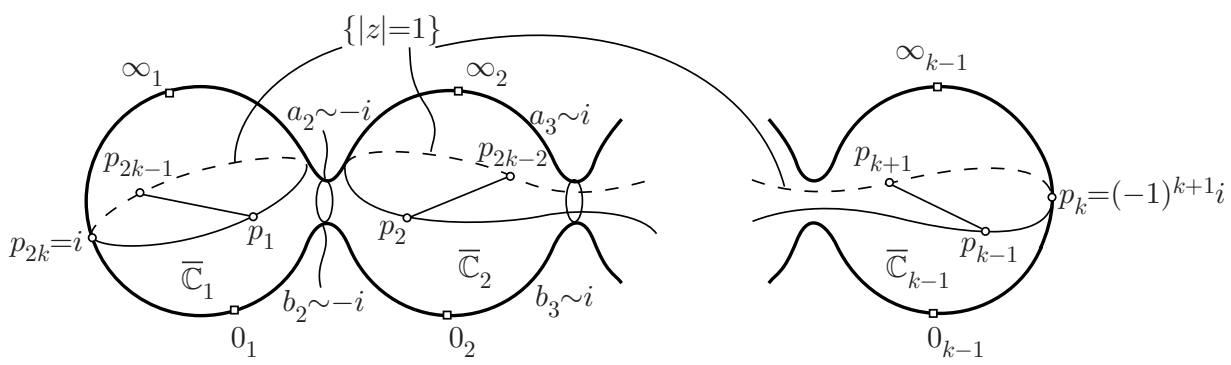

Figure 4. Parametrizing the Gauss map $g$ for the particular case $u \in \mathcal{U}(k)$ close to $\mathbf{u}_{\infty}$. The points $p_{j}, p_{2 k-j} \in\{|z|=1\}$ are respectively close to $\alpha,-\alpha$ in each copy of $\overline{\mathbb{C}}$.

to $(-1)^{j+1} i$. Glue $\overline{\mathbb{C}}_{j-1}$ with $\overline{\mathbb{C}}_{j}$ along a cut from $a_{j}$ to $b_{j}$ in the usual way, so that the chain of $k-1$ copies of $\overline{\mathbb{C}}$ yields a compact surface $\Sigma$ of genus zero. On $\Sigma$ we consider the meromorphic function $g$ defined as $g=z$ in each $\overline{\mathbb{C}}_{j}$. Then $g$ has degree $k-1$ and its branch values are $a_{j}, b_{j}, 1<j<k$. This function $g$ together with the ordered collection of points $p_{1}, \ldots, p_{2 k} \in \Sigma$ given below determine an element of $\mathcal{W}(k)$.

- For $1 \leq j<k, p_{j}$ and $p_{2 k-j}$ are the unique points in $\overline{\mathbb{C}}_{j}$ that satisfy $g\left(p_{j}\right)=p_{j}=(-1)^{j+1} i u_{j}, g\left(p_{2 k-j}\right)=p_{2 k-j}=(-1)^{j+1} i u_{2 k-j}$ (compare with (3.1) $)$. Note that since $\mathbf{u}$ is close to $\mathbf{u}_{\infty}$ and $\alpha \neq \pm 1$, we deduce that $p_{j}, p_{2 k-j}$ are far from the neck between $\overline{\mathbb{C}}_{j-1}$ and $\overline{\mathbb{C}}_{j}$.

- $p_{k}$ lies in $\overline{\mathbb{C}}_{k-1}$ and $p_{2 k}$ in $\overline{\mathbb{C}}_{1}$, are determined by $g\left(p_{k}\right)=p_{k}=(-1)^{k+1} i u_{k}$, $g\left(p_{2 k}\right)=p_{2 k}=-i u_{2 k}$. Again $p_{k}$ is far from the neck between $\overline{\mathbb{C}}_{k-2}$ and $\overline{\mathbb{C}}_{k-1}$, and $p_{2 k}$ is far from the neck between $\overline{\mathbb{C}}_{1}$ and $\overline{\mathbb{C}}_{2}$; see Figure 4

Hence we have defined an element $g \in \mathcal{W}(k)$ from the $4 k-4$ complex parameters $a_{j}, b_{j}, u_{h}$. Since the roles of $a_{j}$ and $b_{j}$ are symmetric, the right parameters to consider if we want to parametrize the space of $g \in \mathcal{W}(k)$ around the Scherk limit are the $u_{h}$ together with the elementary symmetric functions of $a_{j}, b_{j}$. Let

$$
x_{j}=\frac{a_{j}+b_{j}}{2}, \quad y_{j}=a_{j} b_{j}, \quad 1<j<k .
$$

We also exchange the variable $y_{j}$ by $\zeta_{j}=y_{j}-x_{j}^{2}$ for $1<j<k$ (the map $\left(x_{j}, y_{j}\right) \mapsto$ $\left(x_{j}, \zeta_{j}\right)$ is a diffeomorphism). Next we introduce some notation. Given $\varepsilon>0$, let

$$
\begin{aligned}
& \mathbf{x}_{\infty}=\left(-i, i, \ldots,(-1)^{k} i\right), \mathbf{0}=(0 \ldots, 0) \in \mathbb{C}^{k-2} \\
& D\left(\mathbf{x}_{\infty}, \varepsilon\right)=\left\{\mathbf{x} \in \mathbb{C}^{k-2} \mid\left\|\mathbf{x}-\mathbf{x}_{\infty}\right\|<\varepsilon\right\} \\
& D(\mathbf{0}, \varepsilon)=\left\{\zeta \in \mathbb{C}^{k-2} \mid\|\zeta\|<\varepsilon\right\} \\
& D\left(\mathbf{u}_{\infty}, \varepsilon\right)=\left\{\mathbf{u} \in \mathbb{C}^{2 k} \mid\left\|\mathbf{u}-\mathbf{u}_{\infty}\right\|<\varepsilon\right\} \\
& \mathcal{A}=\left\{\zeta \in D(\mathbf{0}, \varepsilon) \mid \zeta_{j}=0 \text { for some } j\right\}
\end{aligned}
$$

Since $\zeta_{j}=-\frac{1}{4}\left(a_{j}-b_{j}\right)^{2}$, the equation $\zeta_{j}=0$ means that $a_{j}=b_{j}$ is a node, so $\mathcal{A}$ expresses when $\Sigma$ pinches into a Riemann surface with nodes. It is easy to show that the map $\Theta: D\left(\mathbf{x}_{\infty}, \varepsilon\right) \times(D(\mathbf{0}, \varepsilon) \backslash \mathcal{A}) \times D\left(\mathbf{u}_{\infty}, \varepsilon\right) \rightarrow \mathcal{W}(k)$ defined by $\Theta(\mathbf{x}, \zeta, \mathbf{u})=g$ is a local chart for $\mathcal{W}(k)$. Given $(\mathbf{x}, \zeta, \mathbf{u}) \in D\left(\mathbf{x}_{\infty}, \varepsilon\right) \times(D(\mathbf{0}, \varepsilon) \backslash \mathcal{A}) \times D\left(\mathbf{u}_{\infty}, \varepsilon\right)$, we call $\phi$ to the height differential associated to $g=\Theta(\mathbf{x}, \zeta, \mathbf{u})$ (see Section 3 ). 
7.2. The equations. Next we study the regularity of the induced metric by the pair $(g, \phi)$. Let $0_{j}, \infty_{j}$ be the points of $\Sigma$ given by $z=0$ and $z=\infty$ in $\overline{\mathbb{C}}_{j}$. The equations we have to solve are $\phi=0$ at $0_{j}$ and $\infty_{j}$ for $1 \leq j<k$ (in which case $\phi$ clearly has necessarily simple zeros at $0_{j}, \infty_{j}$ because $\phi$ has $2 k$ poles in $\Sigma$ ). These equations can be equivalently written as

$$
\operatorname{Res}_{0_{j}}\left(g^{-1} \phi\right)=0, \quad \operatorname{Res}_{\infty_{j}}(g \phi)=0, \quad 1 \leq j<k .
$$

As was explained in Subsection 3.1] we may forget two of these equations by the residue theorem, so we will only solve these equations for $1<j<k$.

We saw in Section 3 that the period problem at the ends writes as $\left|g\left(p_{j}\right)\right|=1$ or equivalently $\left|\operatorname{Res}_{p_{j}}(g \phi)\right|=1$ for $1 \leq j \leq 2 k$ (these equalities are automatically satisfied when the components of $\mathbf{u}$ lie in $\mathbb{S}^{1}$ rather than only in $\mathbb{C}$ ).

Our next goal is to study the behavior of both $g, \phi$ and the equations we have to solve when $\zeta \in \mathcal{A}$. In this case, $\Sigma$ decomposes into a number of spheres between 2 and $k-1, g$ produces nonconstant meromorphic maps on them whose degrees add up to $k-1$ and the values of these meromorphic maps at the node points are $\pm i$.

7.3. Holomorphic extension of $\phi$. Given $(\mathbf{x}, \zeta, \mathbf{u}) \in D\left(\mathbf{x}_{\infty}, \varepsilon\right) \times(D(\mathbf{0}, \varepsilon) \backslash \mathcal{A}) \times$ $D\left(\mathbf{u}_{\infty}, \varepsilon\right)$, let $\gamma_{j}$ be a small circle enclosing the points $a_{j}, b_{j}$ in $\overline{\mathbb{C}}_{j}$, with the positive orientation. By the residue theorem, we have

$$
\frac{1}{2 \pi i} \int_{\gamma_{j}} \phi=\operatorname{Res}_{p_{2 k}} \phi+\sum_{\ell=1}^{j-1}\left(\operatorname{Res}_{p_{\ell}} \phi+\operatorname{Res}_{p_{2 k-\ell}} \phi\right)=(-1)^{j+1} i
$$

When $a_{j}=b_{j}$, the Riemann surface $\Sigma$ has a node at $a_{j}$. In this case the definition of $\phi$ must be changed as follows. For each double point $a_{j}=b_{j}$, we ask that $\phi$ has a simple pole at the point $a_{j} \in \overline{\mathbb{C}}_{j}$ with residue $(-1)^{j+1} i$ (this comes from equation (7.1) ), and it has a simple pole at the point $a_{j} \in \overline{\mathbb{C}}_{j-1}$ with opposite residue.

Proposition 7.1. $\phi$ depends holomorphically on all parameters $(\mathbf{x}, \zeta, \mathbf{u}) \in D\left(\mathbf{x}_{\infty}, \varepsilon\right)$ $\times D(\mathbf{0}, \varepsilon) \times D\left(\mathbf{u}_{\infty}, \varepsilon\right)$ (including those tuples with $\left.\zeta \in \mathcal{A}\right)$. By this we mean that if $z \in \overline{\mathbb{C}}_{j}$ is away from the cuts and poles, then $\phi(z) / d z$ depends holomorphically on $(\mathbf{x}, \zeta, \mathbf{u})$.

Proof. This result is standard; see e.g. Section 3.4 of [19] for Riemann surfaces with nodes and arbitrary genus.

Corollary 7.2. For $1 \leq j<k$, the maps $(\mathbf{x}, \zeta, \mathbf{u}) \in D\left(\mathbf{x}_{\infty}, \varepsilon\right) \times D(\mathbf{0}, \varepsilon) \times$ $D\left(\mathbf{u}_{\infty}, \varepsilon\right) \mapsto \operatorname{Res}_{0_{j}}\left(g^{-1} \phi\right)$, $\operatorname{Res}_{\infty_{j}}(g \phi)$, Res $p_{j}(g \phi)$ are holomorphic.

7.4. The modified ligature map. Let $\widetilde{L}: D\left(\mathbf{x}_{\infty}, \varepsilon\right) \times D(\mathbf{0}, \varepsilon) \times D\left(\mathbf{u}_{\infty}, \varepsilon\right) \rightarrow$ $\mathbb{C}^{4 k-4}$ be the map defined by

$$
\widetilde{L}=(\underbrace{\operatorname{Res}_{0_{j}}\left(g^{-1} \phi\right)}_{1<j<k}, \underbrace{\operatorname{Res}_{\infty_{j}}(g \phi)}_{1<j<k}, \underbrace{\operatorname{Res}_{p_{j}}(g \phi)}_{1 \leq j \leq 2 k}) .
$$

Corollary 7.2 implies that $\widetilde{L}$ is holomorphic. A straightforward computation gives that $\widetilde{L}\left(\mathbf{x}_{\infty}, \mathbf{0}, \mathbf{u}_{\infty}\right)=\left(\mathbf{0}, \mathbf{0}, \mathbf{u}_{\infty}\right) \in\left(\mathbb{C}^{k-2}\right)^{2} \times \mathbb{C}^{2 k}$. 
Lemma 7.3. With the notation above, the Jacobian $(2 k-4)$-matrix

$$
\left.\frac{\partial\left(\operatorname{Res}_{0_{j}}\left(g^{-1} \phi\right), \operatorname{Res}_{\infty_{j}}(g \phi)\right)}{\partial(\mathbf{x}, \zeta)}\right|_{(\mathbf{x}, \zeta, \mathbf{u})=\left(\mathbf{x}_{\infty}, \mathbf{0}, \mathbf{u}_{\infty}\right)}
$$

is regular.

Proof. We start with the partial derivatives of $\operatorname{Res}_{0_{h}}\left(g^{-1} \phi\right)$, Res $\infty_{h}(g \phi)$ with respect to the $x_{j}$ variables. Then we can fix all the $\zeta_{h}$ as zero for $1<h<k$, which means that $\Sigma$ consists of copies $\overline{\mathbb{C}}_{1}, \ldots, \overline{\mathbb{C}}_{k-1}$ of $\overline{\mathbb{C}}$ joined by $k-2$ nodes so that the node between $\overline{\mathbb{C}}_{j-1}$ and $\overline{\mathbb{C}}_{j}$ is placed at $x_{j}=a_{j}=b_{j} \sim(-1)^{j+1} i$ (here $x_{j}$ must be thought as a variable) and the remaining nodes are all placed as $\pm i$. For $h \neq j-1, j$ fixed, the corresponding height differential $\phi$ in $\overline{\mathbb{C}}_{h}$ does not depend on $x_{j}$, hence

$$
\frac{\partial \operatorname{Res}_{0_{h}}\left(g^{-1} \phi\right)}{\partial x_{j}}\left(\mathbf{x}_{\infty}, \mathbf{0}, \mathbf{u}_{\infty}\right)=\frac{\partial \operatorname{Res}_{\infty_{h}}(g \phi)}{\partial x_{j}}\left(\mathbf{x}_{\infty}, \mathbf{0}, \mathbf{u}_{\infty}\right)=0 .
$$

Concerning $\left.\frac{\partial}{\partial x_{j}}\right|_{\left(\mathbf{x}_{\infty}, \mathbf{0}, \mathbf{u}_{\infty}\right)} \operatorname{Res}_{0_{j}}\left(g^{-1} \phi\right)$, the holomorphic extension of $\phi$ lets us write

$$
\phi=(-1)^{j} i\left(\frac{1}{z-p_{j}}+\frac{1}{z-p_{2 k-j}}-\frac{1}{z-x_{j}}-\frac{1}{z-x_{\infty, j+1}}\right) \quad \text { on } \overline{\mathbb{C}}_{j},
$$

where $x_{\infty, j+1}=(-1)^{j} i$ (when $j=k-1$, we should replace $x_{\infty, j+1}$ by $p_{k}=(-1)^{k+1} i$ in the above formula). This gives

$$
\operatorname{Res}_{0_{j}}\left(g^{-1} \phi\right)=(-1)^{j} i\left(\frac{1}{x_{j}}+\frac{1}{x_{\infty, j+1}}\right), \quad \operatorname{Res}_{\infty_{j}}(g \phi)=(-1)^{j} i\left(x_{j}+x_{\infty, j+1}\right),
$$

hence

$$
\frac{\partial \operatorname{Res}_{0_{j}}\left(g^{-1} \phi\right)}{\partial x_{j}}\left(\mathbf{x}_{\infty}, \mathbf{0}, \mathbf{u}_{\infty}\right)=\frac{\partial \operatorname{Res}_{\infty_{j}}(g \phi)}{\partial x_{j}}\left(\mathbf{x}_{\infty}, \mathbf{0}, \mathbf{u}_{\infty}\right)=(-1)^{j} i .
$$

Analogously,

$$
\frac{\partial \operatorname{Res}_{0_{j}}\left(g^{-1} \phi\right)}{\partial x_{j+1}}\left(\mathbf{x}_{\infty}, \mathbf{0}, \mathbf{u}_{\infty}\right)=\frac{\partial \operatorname{Res}_{\infty_{j}}(g \phi)}{\partial x_{j+1}}\left(\mathbf{x}_{\infty}, \mathbf{0}, \mathbf{u}_{\infty}\right)=(-1)^{j} i .
$$

We now compute partial derivatives with respect to $\zeta_{j}$, so we fix all the $x_{h}$ as the corresponding component $x_{\infty, h}$ of $\mathbf{x}_{\infty}, \zeta_{h}=0$ for all $h \neq j$ and think of $\zeta_{j}$ as a variable close to zero. This means that $\Sigma$ decomposes into $k-2$ spheres joined by nodes, $k-3$ of which correspond to single copies of $\overline{\mathbb{C}}_{h}$ (that we will call simple spheres), $h \neq j-1, j$, and just one sphere corresponds to the copies $\overline{\mathbb{C}}_{j-1}, \overline{\mathbb{C}}_{j}$ (called a double sphere). On any simple sphere, neither $g$ nor $\phi$ depend on $\zeta_{j}$, hence

$$
\frac{\partial \operatorname{Res}_{0_{h}}\left(g^{-1} \phi\right)}{\partial \zeta_{j}}\left(\mathbf{x}_{\infty}, \mathbf{0}, \mathbf{u}_{\infty}\right)=\frac{\partial \operatorname{Res}_{\infty_{h}}(g \phi)}{\partial \zeta_{j}}\left(\mathbf{x}_{\infty}, \mathbf{0}, \mathbf{u}_{\infty}\right)=0, \quad h \neq j-1, j .
$$

The remaining double sphere can be parametrized as

$$
S=\left\{(z, w) \in(\overline{\mathbb{C}})^{2} \mid w^{2}=\left(z-a_{j}\right)\left(z-b_{j}\right)=\left(z-x_{\infty, j}\right)^{2}+\zeta_{j}\right\} .
$$

The square root $w=\sqrt{\left(z-x_{\infty, j}\right)^{2}+\zeta_{j}}$ is well defined on $S$, and we fix the sign of this square root as follows. Away from the cut we have $w^{2} \sim\left(z-x_{\infty, j}\right)^{2}$. We ask that $w \sim z-x_{\infty, j}$ in $\overline{\mathbb{C}}_{j}$, and consequently $w \sim-\left(z-x_{\infty, j}\right)$ in $\overline{\mathbb{C}}_{j-1}$. Clearly 
$g$ writes $g(z, w)=z$ on $S$, and an elementary argument using poles and residues leads to

$$
\phi=(-1)^{j} i\left(\frac{w\left(p_{j}\right)}{z-z\left(p_{j}\right)}+\frac{w\left(p_{2 k-j}\right)}{z-z\left(p_{2 k-j}\right)}-\frac{w\left(x_{\infty, j+1}\right)}{z-x_{\infty, j+1}}\right) \frac{d z}{w}
$$

This gives

$$
\begin{gathered}
\operatorname{Res}_{0_{j}}\left(g^{-1} \phi\right)=\frac{(-1)^{j} i}{w(0)}\left(\frac{w\left(p_{j}\right)}{-z\left(p_{j}\right)}+\frac{w\left(p_{2 k-j}\right)}{-z\left(p_{2 k-j}\right)}+\frac{w\left(x_{\infty, j+1}\right)}{x_{\infty, j+1}}\right), \\
\operatorname{Res}_{\infty_{j}}(g \phi)=(-1)^{j} i\left(-w\left(p_{j}\right)-w\left(p_{2 k-j}\right)+w\left(x_{\infty, j+1}\right)\right) .
\end{gathered}
$$

The same formulas with opposite signs hold for the residues at $0_{j-1}$ and $\infty_{j-1}$, because of the valuation of the square root. Using that $x_{\infty, j+1}=(-1)^{j} i$ and $z\left(p_{j}\right)=(-1)^{j+1} i \alpha$ one easily computes

$$
\frac{\partial \operatorname{Res}_{0_{j}}\left(g^{-1} \phi\right)}{\partial \zeta_{j}}\left(\mathbf{x}_{\infty}, \mathbf{0}, \mathbf{u}_{\infty}\right)=\frac{5-\alpha^{2}}{4\left(\alpha^{2}-1\right)}, \quad \frac{\partial \operatorname{Res}_{\infty_{j}}(g \phi)}{\partial \zeta_{j}}\left(\mathbf{x}_{\infty}, \mathbf{0}, \mathbf{u}_{\infty}\right)=\frac{3+\alpha^{2}}{4\left(\alpha^{2}-1\right)}
$$

and the corresponding partial derivatives of the residues at $0_{j-1}$ and $\infty_{j-1}$ only differ from the above ones in a sign. Now the regularity of the Jacobian matrix in Lemma 7.3 follows directly.

Proposition 7.4. Let $\left\{M_{n}\right\}_{n} \subset \widetilde{\mathcal{S}}(k)$ be a sequence converging weakly to a Scherk limit. Then $M_{n} \in \widetilde{\mathcal{K}}(k)$ for $n$ large enough.

Proof. Without loss of generality we can assume that the fluxes $\mathbf{u}_{n}=F\left(M_{n}\right)$ converge to $\mathbf{u}_{\infty}=\left(\alpha,{ }^{(k-1)}, \alpha, 1,-\alpha, \stackrel{(k-1)}{\cdots},-\alpha,-1\right)$ with $\alpha \in \mathbb{S}^{1}-\{ \pm 1\}$. Since $\left\{M_{n}\right\}_{n}$ converges weakly to the Scherk limit associated to this angle $\alpha$, we deduce that for $n$ large enough, the element $g_{n} \in \mathcal{W}(k)$ that corresponds to $M_{n}$ can be represented by $g_{n}=\Theta\left(\mathbf{x}_{n}, \zeta_{n}, \mathbf{u}_{n}\right)$ for certain $\left(\mathbf{x}_{n}, \zeta_{n}\right) \in D\left(\mathbf{x}_{\infty}, \varepsilon\right) \times(D(\mathbf{0}, \varepsilon)-\mathcal{A})$ (with the same notation of this section). Furthermore $\left(\mathbf{x}_{n}, \zeta_{n}\right) \rightarrow\left(\mathbf{x}_{\infty}, \mathbf{0}\right)$ as $n \rightarrow$ $\infty$.

By Lemma 7.3 and the implicit function theorem, there exists $\varepsilon^{\prime}>0$ small such that for any $\mathbf{u} \in D\left(\mathbf{u}_{\infty}, \varepsilon^{\prime}\right)$ there exists a unique pair $(\mathbf{x}(\mathbf{u}), \zeta(\mathbf{u})) \in D\left(\mathbf{x}_{\infty}, \varepsilon^{\prime}\right) \times$ $D\left(\mathbf{0}, \varepsilon^{\prime}\right)$ so that $\widetilde{L}(\mathbf{x}(\mathbf{u}), \zeta(\mathbf{u}), \mathbf{u})=(0,0, \mathbf{u})$. Note that we do not know if some components of $\zeta(\mathbf{u})$ vanish, so the Riemann surface associated to $(\mathbf{x}(\mathbf{u}), \zeta(\mathbf{u}), \mathbf{u})$ might have nodes. But this proves that for any $\mathbf{u} \in D\left(\mathbf{u}_{\infty}, \varepsilon^{\prime}\right)$, there is (locally) at most one marked minimal surface $M(\mathbf{u}) \in \widetilde{\mathcal{S}}(k)$ with $F(M(\mathbf{u}))=\mathbf{u}$. On the other hand, for $\mathbf{u} \in \mathcal{U}(k) \backslash \mathcal{U}_{0}(k)$ there exists exactly one Karcher saddle tower $M_{\mathbf{u}} \in \widetilde{\mathcal{K}}(k)$ with $F\left(M_{\mathbf{u}}\right)=\mathbf{u}$. Hence if $\mathbf{u}_{n}=F\left(M_{n}\right)$ lies in $\mathcal{U}(k) \backslash \mathcal{U}_{0}(k)$ for $n$ large enough, then Proposition 7.4 clearly holds.

It only remains to analyze the case that after passing to a subsequence, $\mathbf{u}_{n} \in$ $\mathcal{U}_{0}(k)$ for all $n$. In this case, we can write $\mathbf{u}_{n}=\left(\alpha_{n},{ }^{(k-1)}, \alpha_{n}, 1,-\alpha_{n},{ }^{(k-1)},-\alpha_{n},-1\right)$ with $\alpha_{n} \rightarrow \alpha$ as $n \rightarrow \infty$. If we take $\zeta_{j}=0$ and $x_{j}(n)=(-1)^{j+1} \alpha_{n}$ for all $j$, then the previous computations in this section give that $\widetilde{L}\left(\mathbf{x}_{n}, \mathbf{0}, \mathbf{u}_{n}\right)=\left(\mathbf{0}, \mathbf{0}, \mathbf{u}_{n}\right)$ for all $n$, where $\mathbf{x}_{n}=\left(x_{2}(n), \ldots, x_{k-1}(n)\right)$. Geometrically this tuple $\left(\mathbf{x}_{n}, \mathbf{0}, \mathbf{u}_{n}\right)$ also represents a Scherk limit, with $k-1$ Scherk surfaces whose fluxes at the ends are \pm 1 , $\pm \alpha_{n}$. In particular, $\left(\mathbf{x}_{n}, \mathbf{0}, \mathbf{u}_{n}\right)$ does not represent a marked surface in $\widetilde{\mathcal{S}}(k)$, hence locally there are not marked surfaces with flux equal to $\mathbf{u}_{n}$, a contradiction. 
We finish this section by proving Proposition 4.3. Note that this proposition was not used to prove that $\widetilde{\mathcal{S}}_{0}(k)$ is empty, so we may assume that this has already been proven (see section (4). Choose an element $\mathbf{u}_{*} \in \mathcal{U}_{0}(k) \backslash\left\{\mathbf{u}_{0}(k)\right\}$. If Proposition 4.3 does not hold, then there exists a sequence $\left\{M_{n}\right\}_{n} \subset \widetilde{\mathcal{S}}(k) \backslash \widetilde{\mathcal{K}}(k)$ such that $\left\{u_{n}=F\left(M_{n}\right)\right\}_{n} \rightarrow \mathbf{u}_{*}$. By Proposition [5.11(B) and since $\widetilde{\mathcal{S}}_{0}(k)$ is empty, $\left\{M_{n}\right\}_{n}$ converges weakly to a Scherk limit. But this contradicts Proposition 7.4.

\section{UNIQUENESS AROUND THE CATENOID LIMIT}

In this section we will study special surfaces close to the catenoid limit and conclude that a sequence of special surfaces cannot converge to the catenoid limit if $k \geq 3$. This was needed in the proof of Proposition 4.1 .

Geometrically, the catenoid limit is the limit behavior of the Scherk limit when $\alpha \rightarrow 1$, with $\alpha$ as in Section 7 If we follow the line of arguments of that section, what goes wrong is that when $\alpha \rightarrow 1$, the poles of $\phi$ converge to the nodes, which is bad to control the limit of $\phi$. For this reason, the geometric setup in this section if quite different from the previous sections. We consider only special surfaces. We rotate the surface so that all ends but two are horizontal. We call the remaining two ends the top and bottom ends. Now $\phi$ has only two poles at the top and bottom ends, and no poles at the horizontal ends. We scale the surface so that the vertical part of the flux on any horizontal section is, up to sign, equal to $2 \pi$. We are now in a situation very similar to Section 7 of [13, and we will follow the same arguments there up to some minor modifications.

8.1. Weierstrass data. We now write a model for the Weierstrass data of a special surface close to the catenoidal limit in the above geometrical setup. Consider $k-1$ copies $\overline{\mathbb{C}}_{1}, \ldots, \overline{\mathbb{C}}_{k-1}$ of the Riemann sphere $\overline{\mathbb{C}}$, and distinct complex numbers $a_{j}, b_{j}$, $1<j<k$, in a punctured neighborhood of 0 if $j$ is odd and of $\infty$ if $j$ is even. Glue $\overline{\mathbb{C}}_{j-1}$ with $\overline{\mathbb{C}}_{j}$ along the cut from $a_{j}$ to $b_{j}$ in the usual way. This produces a Riemann surface $\Sigma$ of genus 0 together with a meromorphic function $g: \Sigma \rightarrow \overline{\mathbb{C}}$ defined by $g=z$ in each $\overline{\mathbb{C}}_{j}$. Its branch values are $a_{j}, b_{j}$ for $1<j<k$.

The horizontal ends are at the $2(k-1)$ points of $\Sigma$ where $g=0$ or $g=\infty$. We call $0_{j}$ and $\infty_{j}$ the points 0 and $\infty$ in $\overline{\mathbb{C}}_{j}$. The bottom and top ends are respectively some points in $\overline{\mathbb{C}}_{1}$ and $\overline{\mathbb{C}}_{k-1}$. We may orient the surface so that the Gauss map at the bottom end is some nonzero complex number $\alpha$ close to 0 (so the bottom end is the point $z=\alpha$ in $\overline{\mathbb{C}}_{1}$ ). If $k$ is odd, then the Gauss map at the top end is some nonzero complex number $\beta$ close to 0 . If $k$ is even, then the Gauss map at the top end is of the form $1 / \beta$ with $\beta \in \mathbb{C}-\{0\}, \beta$ close to 0 (see Figure 5). A priori, we do not impose any relation between $\alpha$ and $\beta$. Of course, both complex numbers are related by flux arguments. We will expose this connection in Subsection 8.2 .

The height differential $\phi$ is defined as the unique meromorphic 1-form on $\Sigma$ with simple poles at the bottom and top ends, and respective residues 1 and -1 . Let $\Gamma_{j}$ be the unit circle in $\overline{\mathbb{C}}_{j}$ with the positive orientation if $j$ is odd and the negative orientation if $j$ is even. All these curves represent the same homology class in $\Sigma$ and $\int_{\Gamma_{j}} \phi=2 \pi i$. 

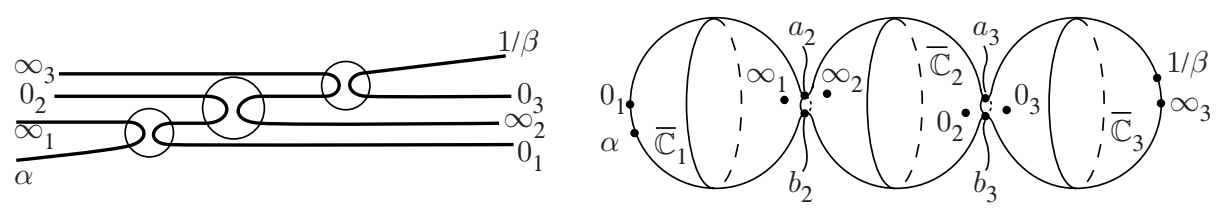

Figure 5. Left: A special surface $M$ close to the catenoid limit in the case $k=4$, with the value of the Gauss map at the ends. Right: The Riemann surface corresponding to $M$, with the ends and the branch points of the Gauss map.

Note that the roles of $a_{j}$ and $b_{j}, 1<j<k$, are symmetric, so the right parameters are their elementary symmetric functions: we introduce the parameters

$$
\begin{aligned}
& x_{j}=\frac{1}{2}\left(a_{j}+b_{j}\right), \quad y_{j}=a_{j} b_{j} \quad \text { if } j \text { is odd, } \\
& x_{j}=\frac{1}{2}\left(\frac{1}{a_{j}}+\frac{1}{b_{j}}\right), \quad y_{j}=\frac{1}{a_{j} b_{j}} \quad \text { if } j \text { is even, }
\end{aligned}
$$

so all parameters $\alpha, \beta, x_{j}, y_{j}, 1<j<k$, are close to 0 . As usual we write $\mathbf{x}=\left(x_{2}, \ldots, x_{k-1}\right)$ and $\mathbf{y}=\left(y_{2}, \ldots, y_{k-1}\right)$.

8.2. The equations. The period problem reduces to impose that the period at each end is the same up to sign. This means that there exists $\lambda \in \mathbb{C}$ such that at each end,

$$
\overline{\operatorname{Res}\left(g^{-1} \phi\right)}+\operatorname{Res}(g \phi)= \pm \lambda .
$$

The period is then $T= \pm \pi(\operatorname{Im} \lambda,-\operatorname{Re} \lambda, 0)$. We also know that the signs \pm alternate at consecutive ends. This gives the following system of equations:

$$
\left\{\begin{array}{l}
\frac{1}{\bar{\alpha}}+\alpha=\lambda, \\
\frac{1}{\bar{\beta}}+\beta= \begin{cases}\lambda & (k \text { odd }), \\
-\bar{\lambda} & (k \text { even }), \\
\operatorname{Res}_{0_{j}}\left(g^{-1} \phi\right)=(-1)^{j} \bar{\lambda}, & 1 \leq j \leq k-1, \\
\operatorname{Res}_{\infty_{j}}(g \phi)=(-1)^{j} \lambda, \quad 1 \leq j \leq k-1 .\end{cases}
\end{array}\right.
$$

Using the residue theorem, these equations imply that

$$
\begin{gathered}
\int_{\Gamma_{j}} g \phi=\int_{\Gamma_{1}} g \phi=2 \pi i \operatorname{Res}_{\alpha}(g \phi)=2 \pi i \alpha \\
\int_{\Gamma_{j}} g^{-1} \phi=\int_{\Gamma_{1}} g^{-1} \phi=2 \pi i\left(\operatorname{Res}_{\alpha}\left(g^{-1} \phi\right)+\operatorname{Res}_{0_{1}}\left(g^{-1} \phi\right)\right) \\
=2 \pi i\left(\frac{1}{\alpha}-\bar{\lambda}\right)=-2 \pi i \bar{\alpha} .
\end{gathered}
$$

Note that $\int_{\Gamma_{j}} g^{-1} \phi$ is conjugate to $\int_{\Gamma_{j}} g \phi$, which means that $\Gamma_{j}$ is a closed curve on the surface, as expected. For $1 \leq j<k$ let

$$
A_{j}= \begin{cases}\frac{1}{2 \pi i} \int_{\Gamma_{j}} g^{-1} \phi & (j \text { odd }) \\ \frac{1}{2 \pi i} \int_{\Gamma_{j}} g \phi & (j \text { even }) .\end{cases}
$$


For $1<j<k$ let

$$
B_{j}= \begin{cases}\operatorname{Res}_{0_{j-1}}\left(g^{-1} \phi\right) \cdot \operatorname{Res}_{0_{j}}\left(g^{-1} \phi\right) & (j \text { odd }) \\ \operatorname{Res}_{\infty_{j-1}}(g \phi) \cdot \operatorname{Res}_{\infty_{j}}(g \phi) & (j \text { even }) .\end{cases}
$$

Then (8.1) implies

$$
\left\{\begin{array}{l}
\beta= \begin{cases}\alpha & (k \text { odd }), \\
-\bar{\alpha} & (k \text { even }),\end{cases} \\
\text { for all } 1 \leq j \leq k-2, \quad A_{j}= \begin{cases}-\bar{\alpha} & (j \text { odd }), \\
\alpha & (j \text { even }),\end{cases} \\
\text { for all } 1<j<k, \quad B_{j}=\left\{\begin{array}{ll}
-\bar{\lambda}^{2} & (j \text { odd }), \\
-\lambda^{2} & (j \text { even }),
\end{array} \quad \text { with } \lambda=\frac{1}{\bar{\alpha}}+\alpha .\right.
\end{array}\right.
$$

In fact, the system (8.1) is equivalent to 8.2), but we will not need that since we want to prove a non-existence result. Note that (8.2) is a system of $2(k-1)$ complex equations in the $2(k-1)$ variables $\alpha, \beta, a_{j}, b_{j}, 1<j<k$. The goal is to prove that for $\alpha \neq 0$ close to 0 , the system (8.2) has no solutions representing a special surface.

8.3. Holomorphic extension of $\phi$. When $a_{j}=b_{j}$ for some $j$, the definition of $g$ gives a Riemann surface with a node between $\mathbb{C}_{j-1}$ and $\mathbb{C}_{j}$. In this case, $\phi$ needs two more simple poles at $z=a_{j}$ in $\mathbb{C}_{j}$ and in $\mathbb{C}_{j-1}$. The residues at these poles are determined by $\int_{\Gamma_{j}} \phi=2 \pi i$. Then as in Section 7 we have

Proposition 8.1. $\phi$ depends holomorphically on $(\alpha, \beta, \mathbf{x}, \mathbf{y})$ in a neighborhood of $(0,0, \mathbf{0}, \mathbf{0})$.

Proof. Same as Proposition 7.1

Proposition 8.2. For $1 \leq j \leq k-2$, the function $A_{j}$ extends as a holomorphic function of $(\alpha, \beta, \mathbf{x}, \mathbf{y})$ in a neighborhood of $(0,0, \mathbf{0}, \mathbf{0})$. The same holds with the function $\widetilde{B}_{j}=y_{j} B_{j}$ for $1<j<k$.

Proof. The first point is a consequence of the previous proposition, since the curve $\Gamma_{j}$ stays in the limit Riemann surface minus its nodes. The second point does not follow from a similar argument because the points $0_{j-1}, 0_{j}\left(\right.$ resp. $\left.\infty_{j-1}, \infty_{j}\right)$ collapse into node points. Instead, we need to control the rate at which the residues in the definition of $B_{j}$ blow up. The reader can find this estimate in the proof of Proposition 10 in [13].

Remark 8.3. $\operatorname{Res}_{0_{j}}\left(g^{-1} \phi\right)$ is a multi-valued function of the parameters. The reason for this is that the points $0_{j}$ and $0_{j-1}$ are close to the branch points $a_{j}, b_{j}$. When the parameters $a_{j}$ and $b_{j}$ vary, the cut from $a_{j}$ to $b_{j}$ may cross 0 , in which case $0_{j}$ and $0_{j-1}$ exchange sheets and so do not depend continuously on the parameters as points on the Riemann surface. On the other hand, the unordered pair $\left\{0_{j-1}, 0_{j}\right\}$ depends continuously on the parameters, which is why the symmetric functions of the residues at $0_{j-1}$ and $0_{j}$ are well-defined functions. This is the main reason why we introduced the functions $B_{j}$. See also Remark 9 in [13]. 


\subsection{Partial derivatives.}

Proposition 8.4. For each $j=2, \ldots, k-1$, it holds that

$$
A_{j-1}(0,0, \mathbf{0}, \mathbf{0})=0, \quad \widetilde{B}_{j}(0,0, \mathbf{0}, \mathbf{0})=-1, \quad \frac{\partial A_{j-1}}{\partial x_{j}}(0,0, \mathbf{0}, \mathbf{0})=1 .
$$

All remaining partial derivatives of the $A_{i}$ with respect to $x_{j}, y_{j}$ are zero. We will not need the partial derivatives of the $\widetilde{B}_{i}$.

Proof. By Proposition 8.2 , for $j$ fixed we can compute either the value of $A_{j-1}, \widetilde{B}_{j}$ or that of their partial derivatives with respect to $x_{j}, y_{j}$ by assuming $\alpha=\beta=0$ and $x_{h}=y_{h}=0$ for all $h \neq j$. The associated Riemann surface has $k-3$ nodes which disconnect it into $k-2$ genus zero components. In each component, the height differential has two simple poles with residue \pm 1 . These poles are either nodes or the top end or the bottom end.

On $k-3$ of these components (which we call simple spheres) the height differential $\phi$ is $d z / z$. The remaining component $S$ corresponds to $\overline{\mathbb{C}}_{j-1}$ glued with $\overline{\mathbb{C}}_{j}$ (we call it a double sphere).

First consider the case $j$ odd. Then $S$ can be parametrized by $\left\{(z, w) \in \overline{\mathbb{C}}^{2} \mid w^{2}=\right.$ $(z-a)(z-b)\}$ where $a+b=2 x_{j}, a b=y_{j}$, so that $w=\sqrt{(z-a)(z-b)}$ is well defined on $S$. We fix the sign of the square root by asking that $w \sim z$ in $\overline{\mathbb{C}}_{j}$ and $w \sim-z$ in $\overline{\mathbb{C}}_{j-1}$. Now $\phi$ has simple poles at $\infty_{j-1}$ and $\infty_{j}$ with respective residues 1 and -1 . As

$$
\operatorname{Res}_{\infty_{j}} \frac{d z}{w}=\operatorname{Res}_{\infty} \frac{d z}{z \sqrt{1-2 x_{j} / z+y_{j} / z^{2}}}=-1,
$$

we conclude that $\phi=d z / w$. Thus

$$
\begin{gathered}
A_{j}=A_{j}\left(x_{j}, y_{j}\right)=\frac{1}{2 \pi i} \int_{\Gamma_{j}} \frac{d z}{z w}=-\operatorname{Res}_{\infty_{j}} \frac{d z}{z w}=0, \\
A_{j-1}\left(x_{j}, y_{j}\right)=\frac{1}{2 \pi i} \int_{\Gamma_{j-1}} \frac{z d z}{w}=\operatorname{Res}_{\infty_{j-1}} \frac{z d z}{-z \sqrt{1-2 x_{j} / z+y_{j} / z^{2}}}=x_{j} .
\end{gathered}
$$

Concerning $\widetilde{B}_{j}$, we write

$$
\operatorname{Res}_{0_{j}}\left(g^{-1} \phi\right)=\frac{1}{\sqrt{y_{j}}}, \quad \operatorname{Res}_{0_{j-1}}(g \phi)=\frac{-1}{\sqrt{y_{j}}} .
$$

The computations in the case $j$ even are similar with the following modifications:

$$
\begin{gathered}
w=\sqrt{\left(\frac{1}{z}-\frac{1}{a}\right)\left(\frac{1}{z}-\frac{1}{b}\right)}, \quad \phi=\frac{-d z}{z^{2} w}, \\
A_{j}\left(x_{j}, y_{j}\right)=\frac{1}{2 \pi i} \int_{\Gamma_{j}} \frac{-d z}{z w}=\operatorname{Res}_{0_{j}} \frac{d z}{z w}=0, \\
A_{j-1}\left(x_{j}, y_{j}\right)=\frac{1}{2 \pi i} \int_{\Gamma_{j-1}} \frac{-d z}{z^{3} w}=\operatorname{Res}_{0_{j-1}} \frac{-d z}{-z^{2} \sqrt{1-2 x_{j} z+y_{j} z^{2}}}=x_{j},
\end{gathered}
$$

which finishes the proof of the proposition. 
8.5. Inverse function theorem. Note that $1 / B_{j}=y_{j} / \widetilde{B}_{j}$. Since $\widetilde{B}_{j}(0,0, \mathbf{0}, \mathbf{0})=$ -1 , the function $1 / B_{j}$ extends holomorphically to a neighborhood of $(0,0, \mathbf{0}, \mathbf{0})$. Moreover

$$
\frac{\partial B_{j}^{-1}}{\partial x_{i}}(0,0, \mathbf{0}, \mathbf{0})=0, \quad \frac{\partial B_{j}^{-1}}{\partial y_{i}}(0,0, \mathbf{0}, \mathbf{0})=-\delta_{i j} .
$$

Define

$$
\Theta(\alpha, \beta, \mathbf{x}, \mathbf{y})=\left(\alpha, \beta, A_{1}, \ldots, A_{k-2}, \frac{1}{B_{2}}, \ldots, \frac{1}{B_{k-1}}\right) .
$$

Then $\Theta$ is holomorphic in a neighborhood of $(0,0, \mathbf{0}, \mathbf{0})$, and its Jacobian matrix at $(0,0, \mathbf{0}, \mathbf{0})$ is invertible. By the inverse function theorem, $\Theta$ is a biholomorphism from a neighborhood of $(0,0, \mathbf{0}, \mathbf{0})$ onto its image. Hence for $t \in \mathbb{C}$ close to 0 , there exists a unique $(\alpha, \beta, \mathbf{x}, \mathbf{y})$ close to $(0,0, \mathbf{0}, 0)$ such that $\alpha=t$ and $\beta, A_{j}, B_{j}$ have the values given by the system (8.2).

We now remark that (8.2) has an obvious solution: For $t \in \mathbb{C}-\{0\}$ take

$$
\alpha=t, \quad \beta=\left\{\begin{array}{ll}
t & (k \text { odd }), \\
-\bar{t} & (k \text { even }),
\end{array} \quad a_{j}=b_{j}= \begin{cases}t & (j \text { odd }), \\
-\frac{1}{\bar{t}} & (j \text { even }) .\end{cases}\right.
$$

The corresponding Riemann surface has $k-2$ nodes which disconnect it into $k-1$ simple spheres. On each sphere $\overline{\mathbb{C}}_{j}$ we have

$$
g=z, \quad \phi=\frac{d z}{z-t}-\frac{d z}{z+\frac{1}{\bar{t}}} .
$$

This is the Weierstrass representation of a singly periodic Scherk minimal surface. It is straightforward to check (and geometrically clear) that (8.2) is satisfied. Geometrically, this solution is a Scherk limit, namely a configuration of $k-1$ Scherk surfaces. It is not a true minimal surface (unless, of course, $k=2$ ). By uniqueness we conclude that (8.2) has no other solutions $(\alpha, \beta, \mathbf{x}, \mathbf{y})$ around $(0,0, \mathbf{0}, \mathbf{0})$, which means that there are no special surfaces close to the catenoid limit if $k \geq 3$.

\section{ACKNOWLEDGMENTS}

Most of the work in this paper was done while the second author was visiting the University of Granada in April 2003. The author would like to thank Antonio Ros for the invitation.

\section{REFERENCES}

1. P. Collin, Topologie et courbure des surfaces minimales de $\mathbb{R}^{3}$, Annals of Math. 2nd Series 145-1 (1997), 1-31. MR1432035 (98d:53010)

2. C. Cosín and A. Ros, A Plateau problem at infinity for properly immersed minimal surfaces with finite total curvature, Indiana Univ. Math. J. 50 (2001), no. 2, 847-879. MR.1871392 (2003d:53013)

3. P. Griffiths and J. Harris, Principles of algebraic geometry (Pure and Applied Mathematics), Wiley-Interscience, 1978. MR0507725 (80b:14001)

4. H. Jenkins and J. Serrin, Variational problems of minimal surface type II. Boundary value problems for the minimal surface equation, Arch. Rational Mech. Anal. 21 (1966), 321-342. MR.0190811 (32:8221)

5. H. Karcher, Embedded minimal surfaces derived from Scherk's examples, Manuscripta Math. 62 (1988), 83-114. MR0958255 (89i:53009)

6. H. Lazard-Holly and W. H. Meeks III, The classification of embedded doubly-periodic minimal surfaces of genus zero, Invent. Math. 143 (2001), 1-27. MR1802791|(2001m:53013) 
7. F. J. López and A. Ros, On embedded complete minimal surfaces of genus zero, J. of Differential Geometry 33 (1991), no. 1, 293-300. MR1085145 (91k:53019)

8. W. H. Meeks III, The theory of triply-periodic minimal surfaces, Indiana Univ. Math. J. 39 (1990), no. 3, 877-936. MR1078743 (92e:53012)

9. W. H. Meeks III, J. Pérez, and A. Ros, Uniqueness of the Riemann minimal examples, Invent. Math. 133 (1998), 107-132. MR 1626477(99b:53013)

10. W. H. Meeks III and H. Rosenberg, The minimal lamination closure theorem, Duke Math J. 133 (2006), no. 3, 467-497. MR2228460

11. 538-578. MR1241472 (95a:53011) 807.53049.

12. - The uniqueness of the helicoid and the asymptotic geometry of properly embedded minimal surfaces with finite topology, Annals of Math. 161 (2005), 723-754. MR2153399 (2006f:53012)

13. J. Pérez, M. Rodríguez, and M. Traizet, The classification of doubly periodic minimal tori with parallel ends, Journal of Diff. Geometry 69 (2005), no. 3, 523-577. MR2170278

14. J. Pérez and A. Ros, Some uniqueness and nonexistence theorems for embedded minimal surfaces, Math. Ann. 295 (1993), no. 3, 513-525. MR.1204835 (94a:53020)

15. - The space of properly embedded minimal surfaces with finite total curvature., Indiana Univ. Math. J. 45 (1996), no. 1, 177-204. MR1406689 (97k:58034)

16. - Properly embedded minimal surfaces with finite total curvature, The Global Theory of Minimal Surfaces in Flat Spaces, Lecture Notes in Math 1775, Springer-Verlag, 2002, G. P. Pirola, editor, pp. 15-66. MR.1901613

17. A. Ros, Embedded minimal surfaces: forces, topology and symmetries, Calc. Var. 4 (1996), 469-496. MR1402733 (98c:53010)

18. H. F. Scherk, Bemerkungen über die kleinsteFläche innerhalb gegebener Grenzen, J. R. Angew. Math. 13 (1835), 185-208, ERAM 013.0481cj.

19. M. Traizet, An embedded minimal surface with no symmetries, J. Differential Geometry 60 (2002), no. 1, 103-153. MR1924593 (2004c:53008)

20. F. Wei, Some existence and uniqueness theorems for doubly periodic minimal surfaces, Invent. Math. 109 (1992), 113-136. MR1168368 (93c:53004)

Departamento de Geometría y Topología, Facultad de Ciencias, Universidad de Granada, 18071, Granada, Spain

E-mail address: jperez@ugr.es

Faculte des Sciences et Techniques, Universite François Rabelais, Parc de GrandMONT, 37200, TOURS, FranCE

E-mail address: martin.traizet@lmpt.univ-tours.fr 